Articles: Discoveries

\title{
The Easter Egg Weevil (Pachyrhynchus) genome reveals synteny in Coleoptera across 200 million years of evolution
}

Matthew H. Van Dam ${ }^{1,3, *}$, Analyn Anzano Cabras ${ }^{2}$, James B. Henderson ${ }^{3}$, Cynthia Pérez

Estrada $^{4}$, Arina D. Omer ${ }^{4}$, Olga Dudchenko ${ }^{4}$, Erez Lieberman Aiden ${ }^{4}$, Athena W. Lam ${ }^{3}$

${ }^{1}$ Entomology Department, Institute for Biodiversity Science and Sustainability, California Academy of Sciences, 55 Music Concourse Dr., San Francisco, CA 94118, USA

${ }^{2}$ Coleoptera Research Center, Institute for Biodiversity and Environment, University of Mindanao, Matina, Davao City, 8000, Philippines

${ }^{3}$ Center for Comparative Genomics, Institute for Biodiversity Science and Sustainability, California Academy of Sciences, 55 Music Concourse Dr., San Francisco, CA 94118, USA.

4

${ }^{4}$ The Center for Genome Architecture, Department of Molecular and Human Genetics, Baylor College of Medicine, Houston, TX 77030, USA

${ }^{*}$ Correspondence to be sent to: Matthew H. Van Dam,

E-mail: matthewhvandam@gmail.com 


\section{The Easter Egg Weevil (Pachyrhynchus) genome reveals synteny in Coleoptera 2 across 200 million years of evolution}

\section{Abstract}

Patterns of genomic architecture across insects remain largely undocumented or decoupled from

7 a broader phylogenetic context. For instance, it is unknown whether translocation rates differ between insect orders? We address broad scale patterns of genome architecture across Insecta by examining synteny in a phylogenetic framework from open source insect genomes. To accomplish this, we add a chromosome level genome to a crucial lineage, Coleoptera. Our

11 assembly of the Pachyrhynchus sulphureomaculatus genome is the first chromosome scale

12 genome for the hyperdiverse Phytophaga lineage and currently the largest insect genome

13 assembled to this scale. The genome is significantly larger than those of other weevils, and this

14 increase in size is caused by repetitive elements. Our results also indicate that, among beetles,

15 there are instances of long-lasting ( $>200 \mathrm{Ma}$ ) localization of genes to a particular chromosome

16 with few translocation events. While some chromosomes have a paucity of translocations, intra-

17 chromosomal synteny was almost absent, with gene order thoroughly shuffled along a

18 chromosome. To place our findings in an evolutionary context, we compared syntenic patterns

19 across Insecta. We find that synteny largely scales with clade age, with younger clades, such as

20 Lepidoptera, having especially high synteny. However, we do find subtle differences in the

21 maintenance of synteny and its rate of decay among the insect orders. 


\section{INTRODUCTION}

Beetles represent one of the most diverse groups of metazoans, with $\sim 400,000$ described species

(Hammond 1992), and estimates of total diversity up to 0.9-2.1 million species (Stork et al.

2015). Among beetles, weevils (Coleoptera: Curculionidae) are one of the most diverse insect

groups (>60,000 species (Oberprieler et al. 2007)), encompassing a huge range of life history

strategies and occupying every conceivable niche in a terrestrial ecosystem. With morphological

31 forms specialized to ecological habits, such as feeding on fungi, seeds, pollen, wood, roots, and even kangaroo dung, weevils make an excellent system in which to study the evolution of different ecomorphologies (Zimmerman 1994, Oberprieler et al. 2007). Weevils belong to the group Phytophaga whose members comprise lineages that specialize on and have co-diversified with many plant lineages (McKenna et al. 2009, Seppey et al. 2019). Given their vast diversity and economic importance as pollinators and crop pests, knowing more about the genomic architecture of beetles should be of broad applicability. However, to date, there are only four available genomes resolved to chromosome level for Coleoptera and none for weevils or the hyperdiverse beetle lineage Phytophaga (Van Belleghem et al. 2018, Fallon et al 2018, Zhang et

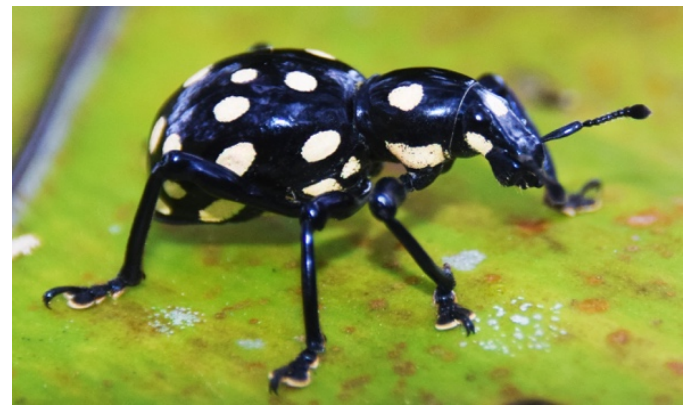

al. 2020, Herndon et al. 2020). Here we present the first genome resolved to chromosome level for the Phytophaga beetle lineage Pachyrhynchus sulphureomaculatus Schultze, 1922.

Figure 1. Pachyrhynchus sulphureomaculatus,

45 lateral habitus. (photo by A. Cabras) 


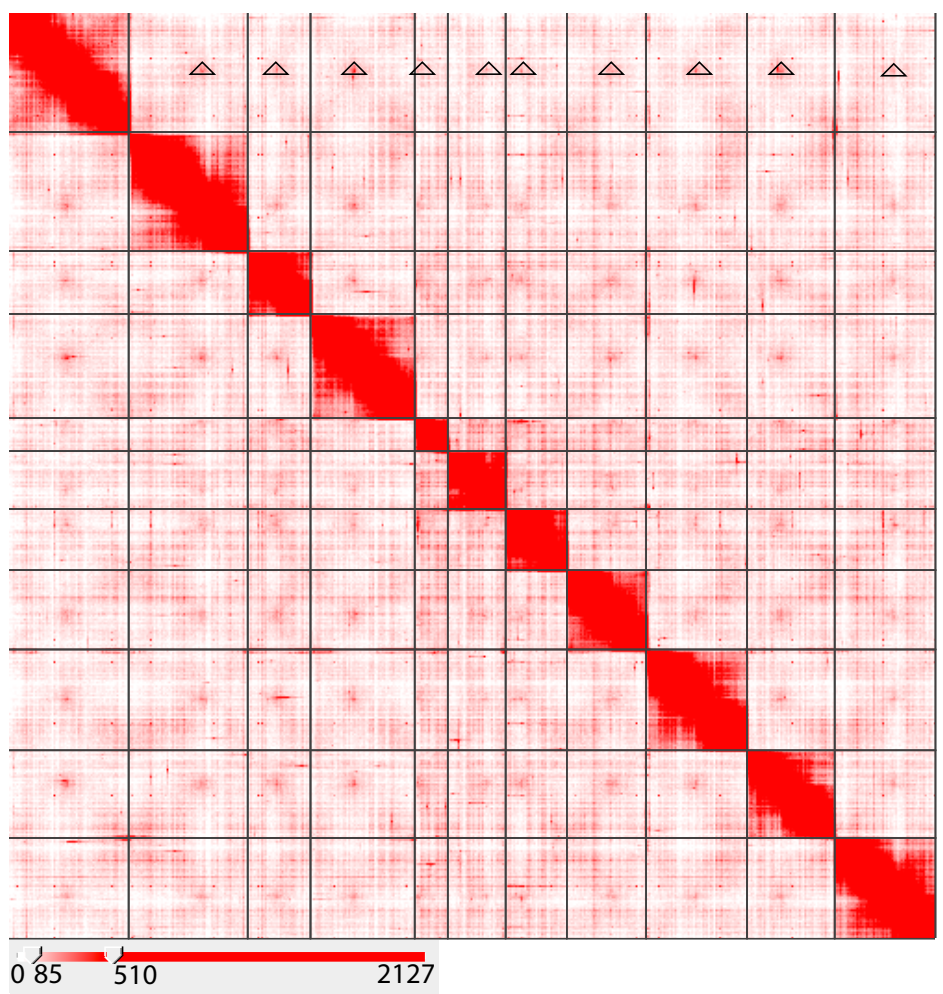

48 Figure 2. Hi-C contact map heatmap of Pachyrhynchus sulphureomaculatus Schultze, 1922. Eleven

49 chromosome boundaries are indicated by black lines. Heatmap scale lower left, range in counts of mapped Hi-

50 C reads per megabase squared. Rabl-like pattern highlighted along chromosome 1, top row, open triangles

51 indicate contact between centromere regions. X-like pattern between adjacent off diagonal regions indicative of contact between distal portions of chromosomes.

54 Recent advances in genome assembly techniques, such as in situ high throughput conformation capture technology (Hi-C) (Lieberman-Aiden et al. 2009), have substantially enhanced our knowledge of genome architecture (Rao et al. 2014, Dudchenko et al. 2017, Eagen et al. 2017).

57 Increasing the accuracy and contiguity of genome assemblies has also been aided by using longread sequencing technology in combination with in situ Hi-C (Ghurye et al. 2017, Matthews et al. 2018, Song et al. 2018, Kingan et al. 2019, Scheffer et al. 2020). These innovations have allowed researchers to not only reconstruct genomes to chromosome scale but also to do so 
61 relatively quickly and cheaply (Dudchenko et al. 2018). In addition, in situ Hi-C technology has

62 shown that the $3 \mathrm{D}$ conformation of genomes is not random and that this conformation can

63 influence gene expression and linkage (Sanborn et al. 2015). The result of these new sequencing

64 techniques has increased the number of high quality genomes for non-model insect species,

65 including beetles (Matthews et al. 2018, Hill et al. 2019, Lu et al. 2019, Liu et al. 2019, Biello et

66 al. 2020, Herndon et al. 2020, Zhang et al. 2020). Because in situ Hi-C orders scaffolds and

67 corrects misjoins, we can study synteny between organisms with more confidence (Dudchenko et

68 al. 2017, Ghurye et al. 2019).

70 With the influx of new chromosome-level genomes, we can now begin to explore patterns of

71 genome architecture within and between major insect lineages. For example, in Lepidoptera

72 (butterflies), genome architecture has been characterized as relatively stable with few (6\%)

73 orthologous loci being translocated (Ahola et al. 2014, Davey et al. 2016, Hill et al. 2019, Wan et

74 al. 2019). Holocentric chromosomes observed throughout Lepidoptera are implicated in reducing

75 hybridization limitations, (Marec et al. 2001, Lukhtanov et al. 2018, Edelman et al. 2019, Hill et

76 al. 2019) suggesting that genome architecture plays a significant role in their biology. In

77 Heliconius butterflies, the inversion and rearrangements that do occur do not seem to hinder

78 hybridization (Edelman et al. 2019). In contrast to Lepidoptera, Drosophila species have many

79 more translocations and rearrangements (Renschler et al. 2019). In beetles, however, even a

80 basic understanding of genomic architecture remains undocumented. The basic blueprints as

81 revealed by in situ Hi-C maps of how a genome is organized (e.g. - with a Rabl-like

82 conformation (Rabl 1885, Csink and Henikoff 1998), holocentric chromosomes, chromosome

83 domain territories, compartments, and topological associated domain loops) remain non-existent 
84 and therefore unplaced in a phylogenetic context. A general synthesis across insects linking these

85 genomic architectural patterns to their function and potential influence on speciation remains

86 incomplete. For example, do different insect orders have distinct rates of genomic

87 rearrangements (the breakage of synteny between genes), or are the patterns we observe merely

88 due to clade age? Are there aspects of a lineages' genomic architecture that contribute to their

89 observed syntenic patterns? Here we address these questions and provide a new chromosome-

$90 \quad$ level genome for Coleoptera.

91

92 RESULTS

93 Sequencing and assembly results

94 From our PacBio library we sequenced a total of $87,452,300,317$ base pairs (bp) with an

95 N50 read length of 31,404 bp (see Supplemental Information Table 1 for full report). From our in

96 situ Hi-C library (we refer to the in situ Hi-C library or reads as "Hi-C" throughout), we

97 sequenced a total of 228,169,567 paired reads after cleaning. Only $2.53 \%$ of our Hi-C reads were
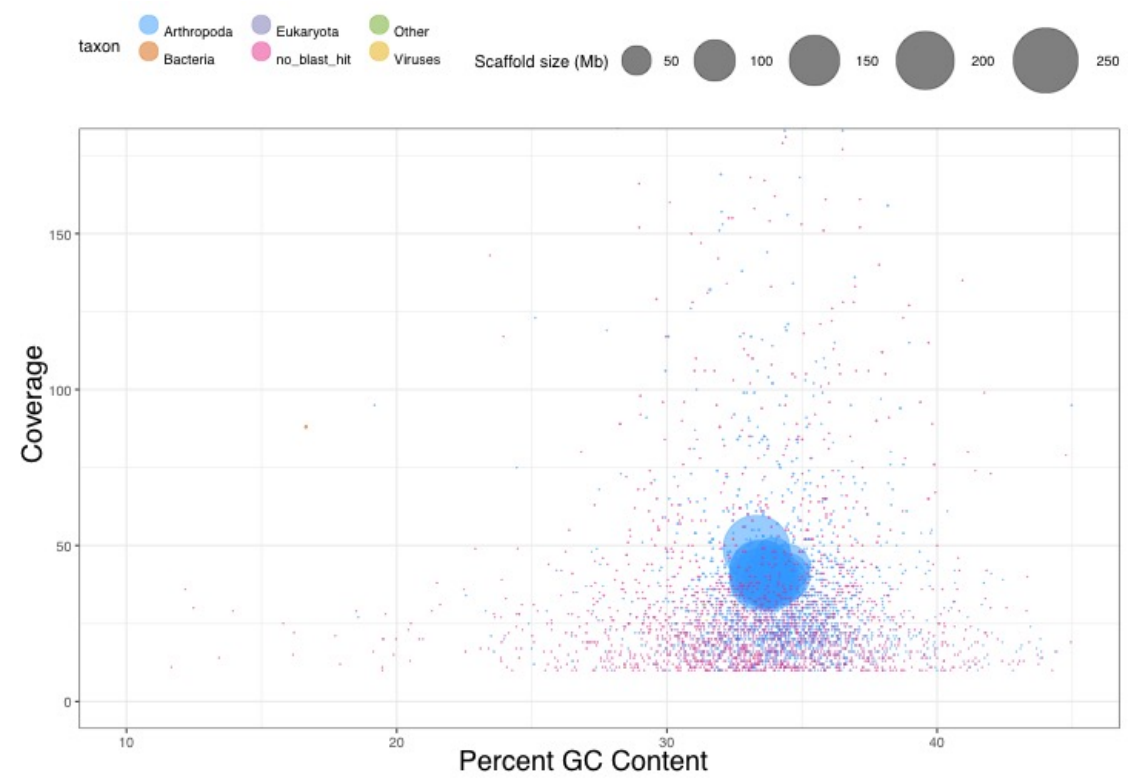

98 Figure 3. P. sulphureomaculatus scaffold bubble plot of coverage versus GC content. Scaffolds included are

99 from the unfiltered assembly. Taxonomic annotation provided via blastn alignment to the NCBI nt database. 
100 unmapped, and we had a total of $80,652,881 \mathrm{Hi}-\mathrm{C}$ contacts. For a list of the intra-/inter-

101 chromosomal contacts and long/short range Hi-C contacts, see Table 1.

102 Our initial assembly after 3X polishing in RACON (Vaser 2017) consisted of 18,240

103 scaffolds and was 2,982,578,979 bp in total length. After removing duplicate haplotigs with

104 Purge Haplotigs (Roach et al. 2018), 9,751 scaffolds and 2,052,097,903 bp remained (Fig. 2).

105 Our initial Hi-C assembly resulted in 4,111 and 2,057,226,403 bp total. The size increase is due

106 to $500 \mathrm{bp}$ insertions of Ns (the 3D-DNA default), between scaffolds merged into super-scaffolds.

107 Running Pilon (v. 1.23) (Walker et al. 2014) in “--fix bases" mode and removal of mitochondrial

108 and contaminant scaffolds (virus or bacteria) resulted in 4,093 scaffolds and 2,051,389,195 bp. A

109 bubble plot of scaffolds by taxon category, and a table of the chromosomes scaffold N50s are

110 shown in Fig. 3 and Table 2, respectively. The identity of other scaffolds not included in the

111 main chromosomes are ambiguous (14 potential viruses and 31 potential bacteria). We retained

112 these but did remove any with bacteria or virus as their best blast score those previously

113 mentioned. The full summary statistics of our final assembly are shown in Table 2 . From the

114 different versions of BUSCO (Felipe et al. 2015) Insecta gene sets (1658 BUSCOs version 2,

1151367 version 4 and 5-beta), the percentage of complete genes varied $(90.8 \% \mathrm{~V} 2,87.6 \% \mathrm{~V} 4$ and

$11691.1 \%$ V5 (Fig. 4)), indicating a relatively complete assembly. Compared to other chromosome-

117 level beetle genomes, we found a comparable number of complete BUSCO genes. However, the

118 results vary somewhat depending on which version of BUSCO and which genes were used (Fig.

119 4). We found a relatively low duplication rate compared to that found in two other beetle

120 (Photinus firefly and Propylea ladybeetle) genomes that used primarily long-read and Hi-C

121 sequencing in their assembly. 

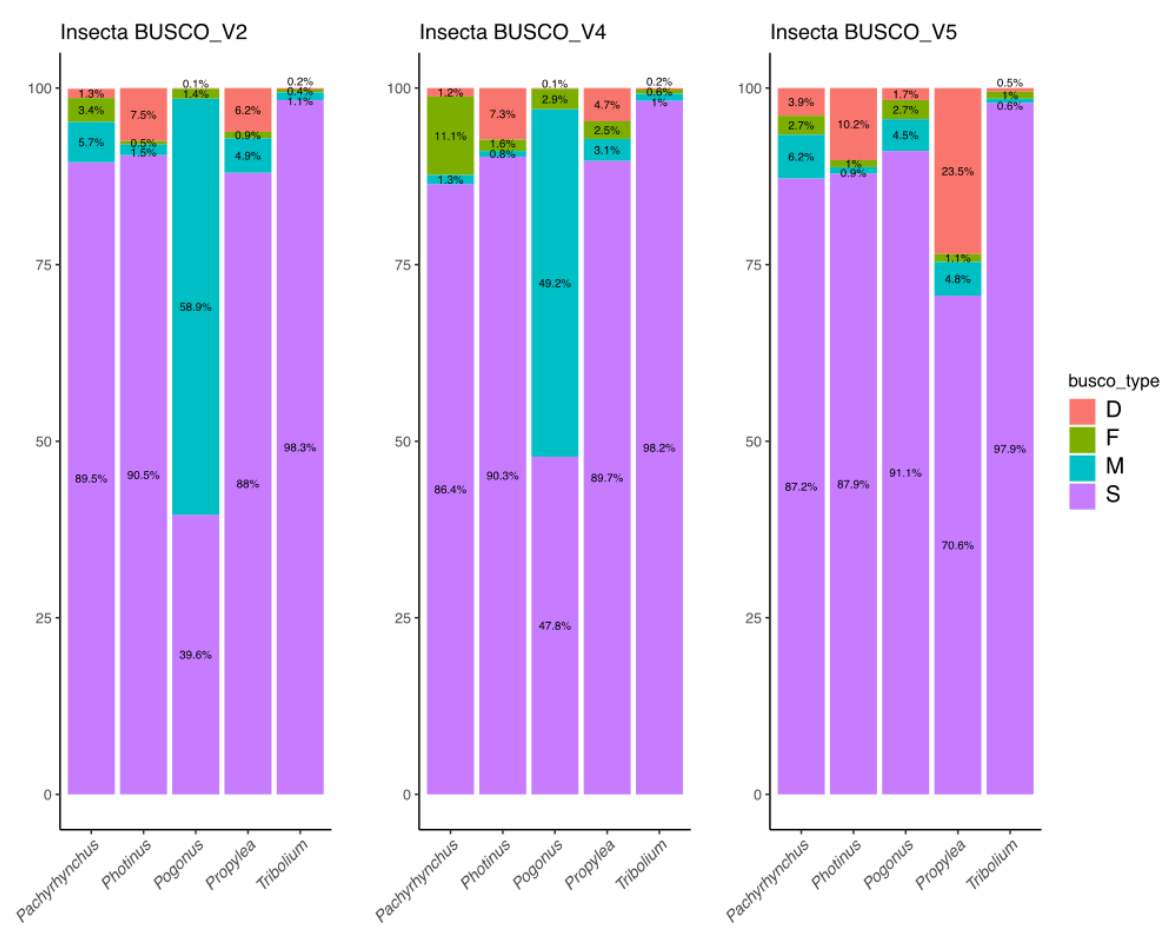

122 Figure 4. Stacked bar plot of Insecta BUSCO gene sets by category for chromosome-level beetle genomes. Y-

123 axis is the percent of BUSCO genes, $\mathrm{X}$-axis labels are the genus names. The abbreviations in the legend are:

$124 \mathrm{D}=$ duplicated, $\mathrm{F}=$ fragmented, $\mathrm{M}=$ missing and $\mathrm{S}=$ single.

Repeat content analyses

127 At $2.05 \mathrm{Gbp}$, the Pachyrhynchus sulphureomaculatus genome is roughly 1.8 times as large as the 128 next largest weevil (Curculionoidea) genome published to date, the $1.11 \mathrm{Gbp}$ Listronotus

129 bonariensis, the Argentine Stem Weevil (Harrop et al. 2020), and 2.6 times the next largest, the 130782 Mbp Red Palm Weevil, Rhynchophorus ferrugineus (Hazzouri et al. 2020) genome. To help 131 explain the size difference, we categorized the repeat content of $P$. sulphureomaculatus. The 132 repeat content analyses from RepeatMasker shows that the genome of $P$. sulphureomaculatus 133 consists of more than three quarters $(76.36 \%)$ repetitive DNA, similar to the repeat percentage of 134 Listronotus, which is the closest relative to Pachyrhynchus. Compared to other weevil genomes

135 (Fig. 5), P. sulphureomaculatus has roughly the same percentage of non-repetitive DNA as 
137 Scolytinae (Dendroctonus and Hypothenemus), are $\sim 1 / 12$ the size of P. sulphureomaculatus and

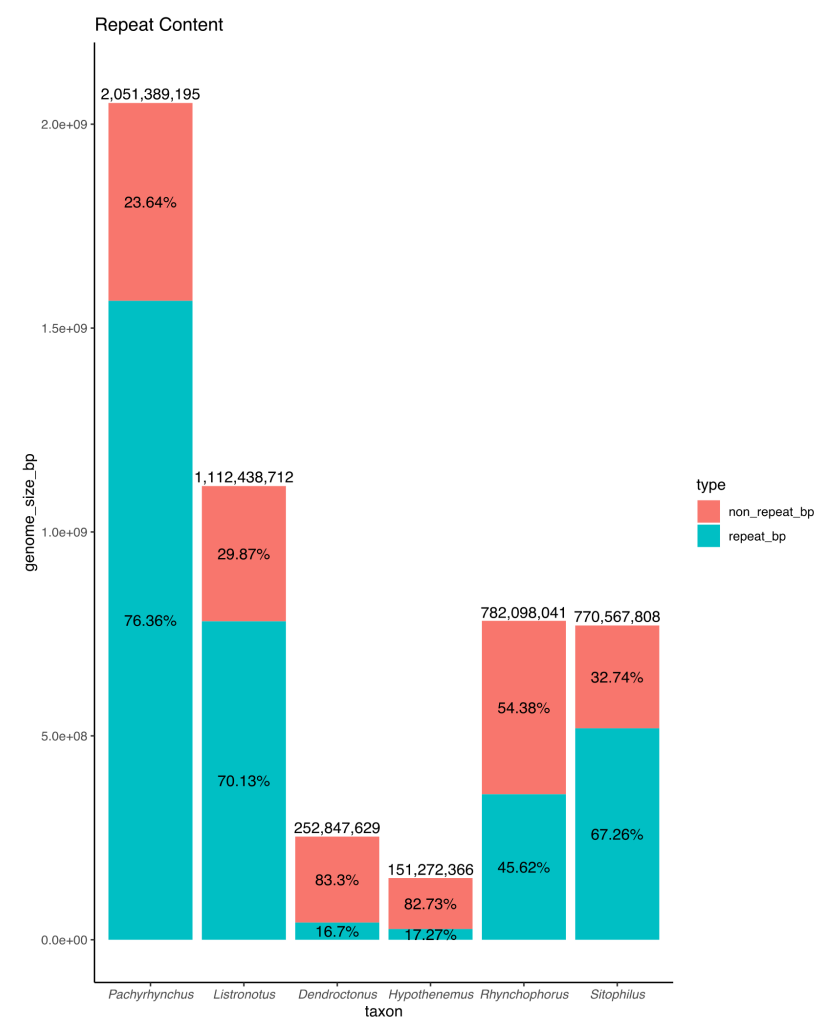
consist of only $\sim 17 \%$ repetitive content. The P. sulphureomaculatus genome consisted of $73.1 \%$ interspersed repeats, with SINEs being 0.1\%, LINEs 20.8\%, LTR elements 2.6\%, DNA elements 33\% and unclassified repeats $16.6 \%$. A sliding window analysis suggests that repetitive content tends to be found in a higher percentage towards the ends of the chromosomes in P. sulphureomaculatus, except in chromosome 5 (Fig. 6).

Figure 5. Histogram of repeat content for weevil genomes.

\section{Genome annotation}

After removing low quality reads from our transcriptome library, a total of 20,551,938

153 paired reads remained. Our initial 3 transcriptome assemblies, Trinity de novo, Trinity genome 154 guided assembly and rnaSPAdes, resulted in fairly similar assemblies, with each having a high 155 number $(\sim 90 \%)$ of the BUSCO v.2 Arthropoda genes (see Supplemental Information Table 2 for 156 details).

As the nuclei of cells between different species generally do not interact (except for 158 viruses), and because Hi-C mapping will remove any non-Pachyrhynchus DNA from the 159 chromosomes, we only annotated genes found within the 11 chromosomes comprising 
161 contained, as percentages of length, $26.00 \%$ genes, $1.46 \%$ exons and $24.54 \%$ introns, with

16230,175 genes, totaling $520,120,665$ bp with a mean length of $17,236.81$. There are a total of

16310,009 single exon genes (33.17\% of genes), a total of 120,454 exons with a mean of 3.99 exons

164 per gene, a length of $242.1 \mathrm{bp}$ and per gene length of $966.41 \mathrm{bp}$. There are a total of 90,279

165 introns with a mean of 2.99 per gene, a length of 5,438.24 bp and a per gene length of 16,270.4

166 bp. The distribution of gene, exon and intron sizes can be found in the Supplemental Information

167 file "Size_of_Genes_Exons_Introns". The gff and tRNA annotations are also in the

168 Supplemental Information. Chromosome gene distribution is relatively even, with only a few

169 regions enriched with genes (Fig. 6).

Synteny across coleopteran chromosome-level genomes

172 We found BUSCO v.2 loci (1658 Insecta gene set) had a low level of translocations between

173 chromosomes (Fig. 7). Our UCE set resulted in 295 loci among taxa and recovered an identical

174 topology and similar dates as in McKenna et al. (2019). We also found a similar synteny pattern

175 between BUSCO genes and those from our UCE set (Supplemental Information file

176 "BUSCO_UCE_chromosome_Tcas_Psulph"). Results show that within a chromosome, the order

177 of BUSCO genes is not conserved (Fig. 7), with few long segments of synteny within a

178 chromosome. Synteny is greatest between P. sulphureomaculatus and the three Polyphaga

179 beetles, and least between Adephaga (Pogonus) and P. sulphureomaculatus. Interestingly, there

180 is more synteny between P. sulphureomaculatus and Photinus pyralis (firefly) (Fallon et al.

181 2018) than between P. sulphureomaculatus and Propylea japonica (ladybird beetle), the closer 
182 relative of the two, indicating that the lineage leading to Propylea has undergone many more

183 chromosomal translocation events (Fig. 7 and 8).

184 We computed the Ensemble Gene Order Conservations (GOC) scores (ref) across all

185 pairwise comparisons for our 34 taxa; results are in Supplemental Information file

186 “GOC_results_matrix.txt”. We recovered 1356 BUSCO Genes for the $70 \%$ complete matrix,

187 totaling 546,311 amino acids in length. The phylogeny recovered the same clades as in Misof et

188 al. (2014). The GOC scores tended to vary with phylogenetic distance between taxa. For

189 example, the hemipterans Triatoam rubrofasciata and Rhodnius prolixus (Reduviidae) scored

190 0.73, and between R. prolixus and the pea aphid Acyrthosiphon pisum, 0.01. Results from the

191 Mantel test between phylogenetic distance and GOC score found that the two variables were

192 correlated $(\mathrm{P} \leq 0.001)$.
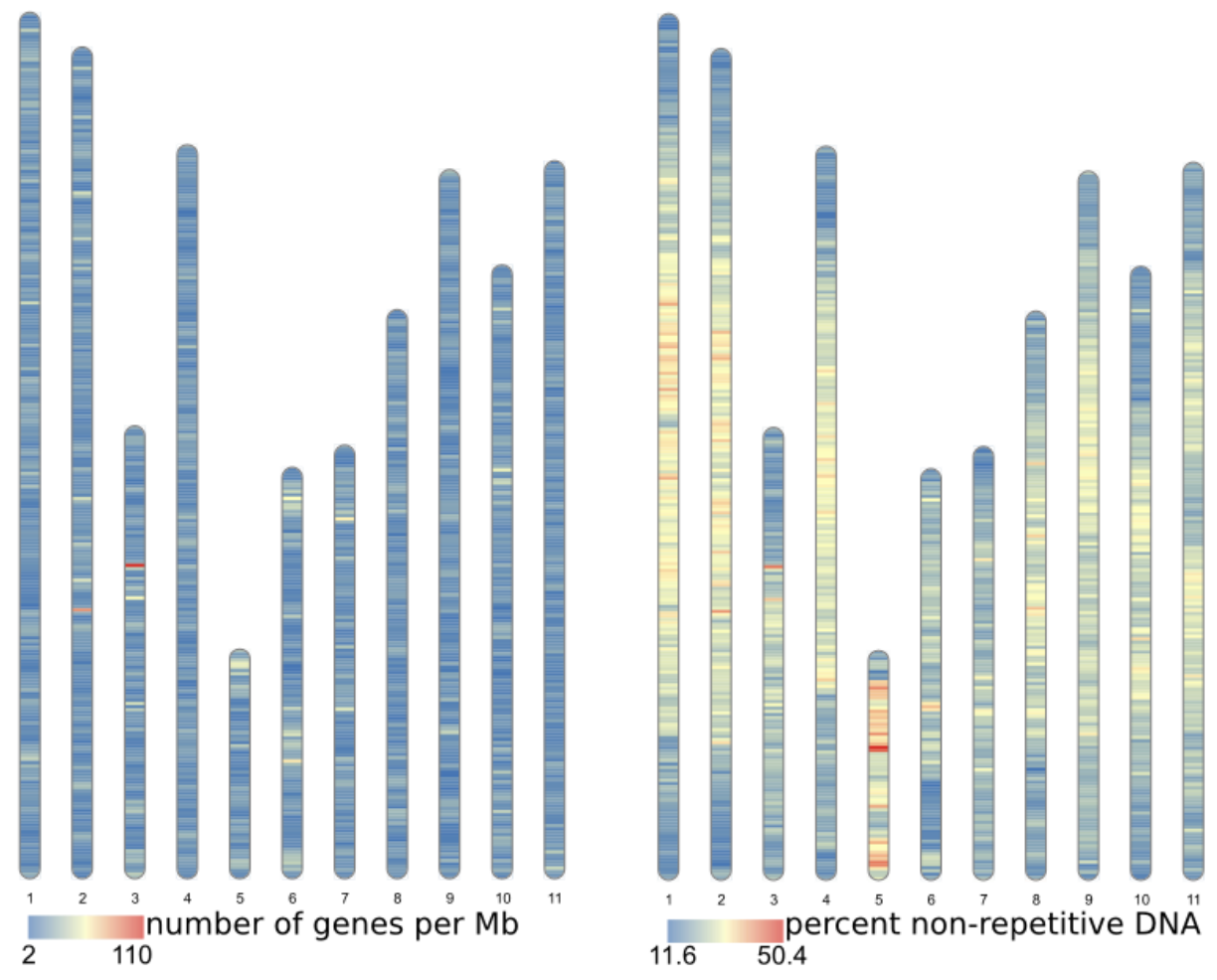

194 Figure 6. Heat map of gene density and non-repetitive DNA per $1 \mathrm{Mb}$ sliding window. The 11 chromosomes 195 are in the same order as in the Hi-C heat map (Fig. 1) and fasta file of the genome. 


\section{DISCUSSION}

The combination of long-read DNA and Hi-C sequencing was successful in resolving a

200 large and highly repetitive insect genome. To date, this is the largest insect genome and one of

201 the largest arthropod genomes assembled to chromosome scale, the horseshoe crab's (Tachypleus

202 tridentatus) being only slightly larger (2.06 Gb vs $2.05 \mathrm{~Gb}$ ) (Zhou et al. 2020). This is

203 remarkable because the assembly of relatively large and highly repetitive insect genomes into

204 highly contiguous ones such as this was previously unattainable ( $\mathrm{Li}$ et al. 2020). Those efforts

205 were hindered by repetitive contents breaking scaffolds or misjoining them (Dudchenko et al.

206 2017, Hill et al. 2019, Li et al. 2020). The unusually large size of the Pachyrhynchus genome is

207 mostly due to the inflated proportion of repetitive content, 76.4\% of the genome (Fig. 5). Again,

208 highlighting the need for long sequencing reads to span the repetitive content. Here we used a

209 single individual to create both our Hi-C and PacBio libraries. The main advantage over using

210 multiple individuals is little loss of $\mathrm{Hi}-\mathrm{C}$ reads mapped to the scaffolds; it also eliminates the

211 need for isogenic lines to be established before sequencing. In our previous attempts to assemble

212 a genome for Pachyrhynchus, we were greatly hindered by the loss of mappable reads when

213 using multiple individuals. As long read sequencing improves in its capabilities of using a small

214 amount (5-50 ng) of DNA, capitalizing on this combination of Hi-C and long-read sequencing

215 will make it feasible to assemble chromosome scale genomes from single, very small insect

216 specimens (Kingan et al. 2019, Schneider et al. 2020). 
bioRxiv preprint doi: https://doi.org/10.1101/2020.12.18.422986; this version posted December 21, 2020. The copyright holder for this preprint (which was not certified by peer review) is the author/funder, who has granted bioRxiv a license to display the preprint in perpetuity. It is made available under aCC-BY-NC-ND 4.0 International license.

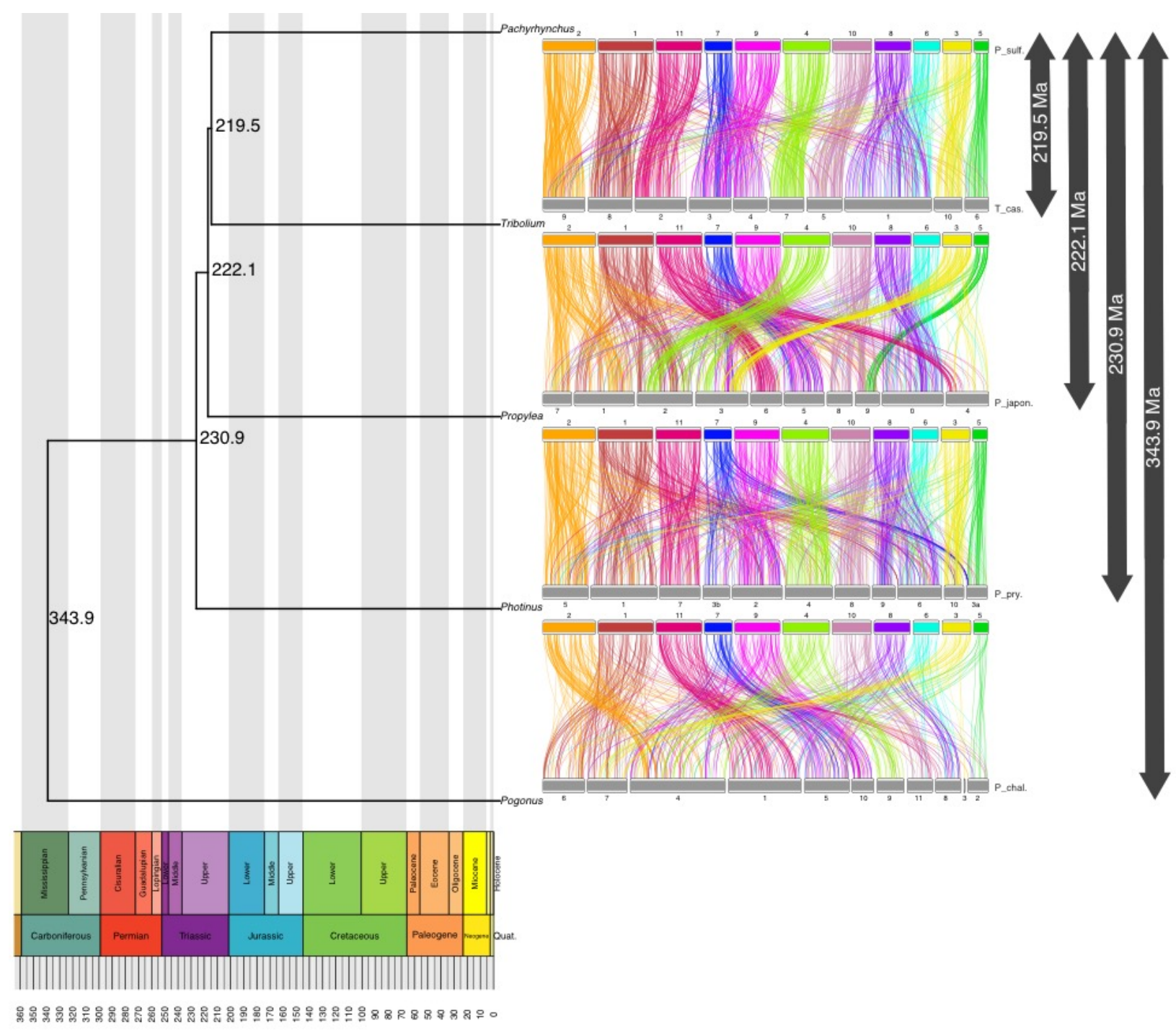

Figure 7. Chronogram and ideograms of the 5 beetle genomes which have chromosome level assemblies. 

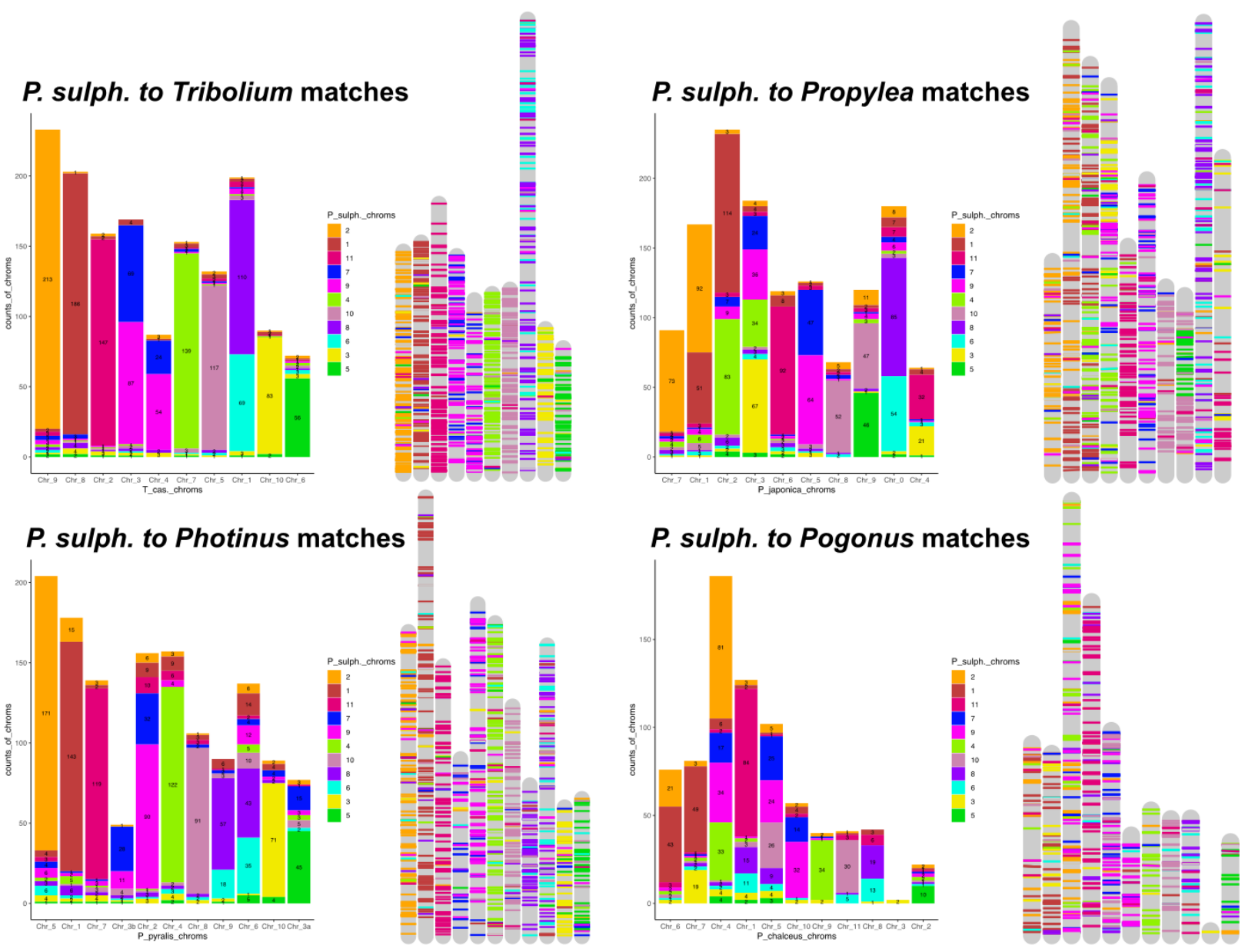

222 Figure 8. Stacked bar plots and chromosome mappings of BUSCO genes' placements. Colors correspond to $P$.

223 sulphureomaculatus chromosomes. The numbering scheme of chromosomes matches the names found in the

224 genome's fasta file.

226 The conserved inter-chromosomal synteny (few chromosome translocations) between the beetle

227 genomes is surprising given the divergence times of the different lineages. For example, we

228 recovered chromosomes that have remained 80-92\% intact for more than $200 \mathrm{Ma}$ (Figs. 7-8). By

229 contrast, the order of the BUSCO genes inside of the chromosomes are highly rearranged, such

230 as chromosomes 8 and 6 in Pachyrhynchus and chromosome 1 in Tribolium (Figs. 7-8). This

231 initial finding prompted us to examine whether similar patterns are observed across other insect

232 orders. A characteristic of Lepidoptera is having a high level of synteny across different families 
233 (Hill et al. 2019, Wan et al. 2019). When we examine the ages or relative branch lengths between

234 clades, we find that much of the synteny is correlated with clade age (Fig. 9), as well as from the

235 results of the Mantel test $(\mathrm{P} \leq 0.001)$. Although Lepidoptera tends to exhibit more synteny given

236 the phylogenetic distance between taxa, there are a few examples other than Drosophila in this

237 same time period to compare against (Fig. 9). Currently, chromosome-level genomes are not

238 available for Trichoptera (caddisflies, the sister lineage to Lepidoptera) or early diverging

239 lineages of Lepidoptera. With the addition of these lineages, we could determine whether the

240 observed pattern of synteny conservation is found only in Lepidopteran crown groups or whether

241 it is more widely dispersed across the entire Lepidopteran lineage. Another order with a

242 somewhat similar level of synteny as in Lepidoptera given their divergence times are Hemiptera

243 (Fig. 9), although there are only 2 comparisons with the same level of divergence (Fig. 9). Taxa

244 with much less synteny given their divergence times are Hymenoptera and Diptera (Fig. 9). The

245 finding that synteny tends to decay with age is not surprising; however, there are some insect

246 orders that are more or less syntenic than expected given their age. For example, in Drosophila,

247 there is less synteny between members of this genus ( $\sim 40 \mathrm{Ma})$ than across all of Lepidoptera or

248 the Aphidae that we examined. These results of gene order conservation are consistent with

249 research of Drosophila topological associated domains (TADs) that showed synteny break points

250 at approximately every 6th gene between D. melanogaster, D. virilis and D. busckii, which have

251 a similar level of divergence as the Drosophila taxa we examined, about 40 Ma of divergence

252 (Renschler et al. 2019). In addition, the chromosomal rearrangement across Drosophila tends to

253 occur at TAD boundaries, not inside the loops (Renschler et al. 2019, Liao et al. 2020). In

254 Anopheles mosquitos, the TAD structures seem to be associated with cytological structures as

255 well (Lukyanchikova et al. 2020). 
257 the boundaries between TADs (Renschler et al. 2019, Liao et al. 2020, Lukyanchikova et al.

258 2020). Despite having many breakpoints, with relatively few chromosome translocations, the

259 dipteran chromosomes largely remain intact (Bracewell et al. 2019). In Coleoptera we find a

260 somewhat similar syntenic pattern, in that the chromosomes remain intact while also being

261 highly shuffled (Figs. 7-8). Given the divergence time between our taxa, when translocations do

262 occur, their initial positions are lost due to a high level of reorganization producing a pattern of

263 interwoven segments. For example, chromosomes 8 and 9 in Pachyrhynchus and the large

264 chromosome 1 in Tribolium (Fig. 8), there are no large syntenic runs of genes or obvious places

265 of translocation. In contrast, chromosome 9 of Propylea and chromosome 5 of Pachyrhynchus

266 are still largely intact, with the homologous segment of chromosome 5 inserted into roughly the

267 middle of Propylea's chromosome 9. Across the Coleoptera we examined, this was the only

268 fusion event that could be roughly placed. Given the relative amount of reshuffling along other

269 parts of this chromosome, the ability to place the insertion indicates that this was a relatively

270 recent event. This large amount of reshuffling within chromosomes with few inter-chromosomal

271 events contrasts with patterns seen in mammals in which the chromosomes tend to exchange

272 larger blocks of material more readily (Chowdhary et al. 1998, Kemkemer et al. 2009, Deakin

273 2018, Simison et al. 2020). 


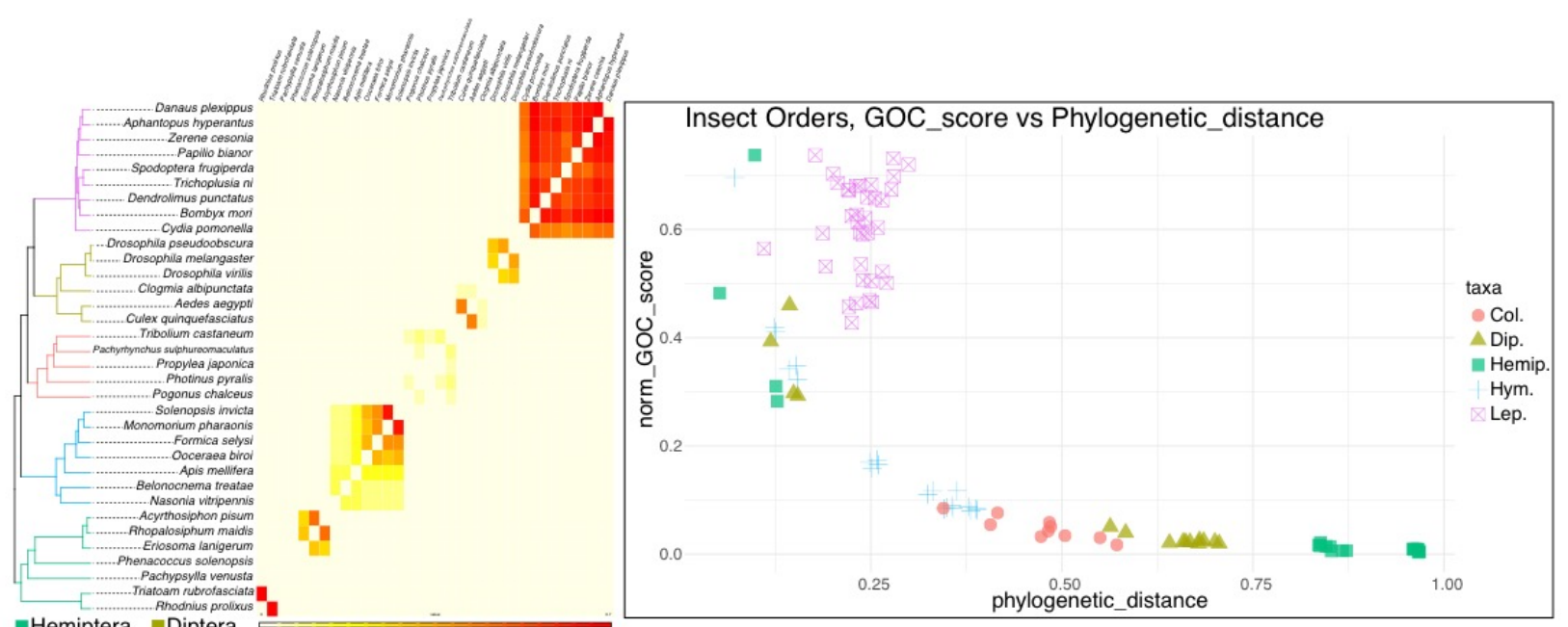

Figure 9. Insecta, gene order conservation score (GOC) plot. Left panel, chronogram, branches colored by order. Heat map of normalized pairwise GOC scores, redder boxes indicate more synteny between pairs. Right panel, within order normalized pairwise GOC score versus phylogenetic distance.

281 includes the Rabl-like configuration of chromosomes, where centromeres and telomeres cluster at opposite/different regions of the cell. These features are important to note because they may serve an important evolutionary function, such as reducing chromosomal entanglements during

284 interphase as well as regulating chromosomal compartmentalization (Mizuguchi et al. 2015,

285 Pouokam et al. 2019). Both major lineages of Diptera, the Nematocera (e.g. mosquitoes and

286 Psychodidae) and Schizophora (e.g. Drosophila), have cells with a Rabl-like configuration

287 (Csink and Henikoff 1998, Dudchenko et al. 2017, Matthews et al. 2018). These taxa span much

288 of the phylogenetic distance across the dipteran lineage, and thus this pattern of chromosomal

289 organization may be characteristic of Diptera. We also observe the Rabl-like configuration in

290 Pachyrhynchus as well as in the Hi-C map of Tribolium (DNAZoo Consortium et al. 2020). Hi-

291 C map observations for the other taxa do not indicate any other obvious cases of the Rabl-like 
configuration within the Insecta. However, improving the quality of existing Hi-C maps would

293 provide more evidence for this observation because a lack of valid Hi-C reads can obscure this

294 type of chromosomal architecture.

295 The Rabl-like configuration is not restricted to beetles and flies; it is also found in the

296 yeast genome (Jin et al. 1998, Goto et al. 2001, Mizuguchi et al. 2015, Kim et al. 2017) as well

297 as in wheat, barley and Brassica (Santos and Shaw 2004, Mascher et al. 2017, Concia et al.

298 2020, Wang et al. 2019), and was originally described from salamander cells (Rabl 1885). It is

299 unclear how widespread the Rabl-like configuration is in Coleoptera. The Hi-C maps of the other

300 beetle genomes do not display this formation and are from similar tissue types to what we used

301 (Fallon et al. 2018). It could be that this configuration is only in the Tenebrionoidea and

302 Phytophaga lineages, where it is presently observed. It is assumed that the Rabl-like

303 configuration is found in all life stages, as appears to be the case in Diptera (Dudchenko et al.

304 2017, Matthews et al. 2018, Lukyanchikova et al. 2020). Additionally, while the Rabl-like

305 configuration is found in different life stages (egg, larva, adult) of Drosophila, it is found

306 intermittently in cells, e.g. found in Drosophila larvae early G1 and not in late G1 interphase of

307 mitosis (Csink and Henikoff, 1998). Moreover, some organisms' cells possess a Rabl-like

308 configuration more often during mitosis as compared to other organisms (Idziak et al. 2015).

309 Therefore, what is visualized on the Hi-C map is not that all cells possess the Rabl-like

310 configuration all the time; instead, it is an average of the occurrence in a particular organism's

311 cells. While the Rabl-like configuration is the predominant chromosomal arrangement observed

312 thus far in Diptera and some Coleoptera, its evolutionary significance remains unclear. Genomic

313 architecture's influence on diversity, if any, is hindered by the sparse, haphazard sampling of

314 insect genomes. It may be tempting to ascribe patterns to clades (such as Lepidoptera being 
315 highly conserved in their genome architecture), but such patterns fade in a broader phylogenetic

316 context or remain to be fully tested. Rather than one to one comparisons, it is more meaningful to

317 describe patterns for a clade in a broader phylogenetic context.

318 In summation, we have reconstructed one of the largest and most repetitive arthropod

319 genomes. With the combination of $\mathrm{Hi}-\mathrm{C}$ reads and PacBio long-read sequencing data, we were

320 able to resolve a highly contiguous, chromosome-level genome. We find patterns of genomic

321 architecture, specifically, synteny across Insecta, largely scales with clade age, with some

322 groups, such as Lepidoptera and Diptera, showing subtly different patterns.

\section{METHODS}

\section{Taxon selection and natural history}

329 Philippines to Papua New Guinea, Australia, Taiwan, Japan, and Indonesia (Schultze, 1923;

330 Alonso-Zarazaga \& Lyal, 1999). They are known for their bright, iridescent and unique elytral

331 markings, which they use as an aposematic signal to warn predators of their unpalatability

332 (Tseng et al., 2014). Members of other weevil groups (e.g. Polycatus, Eupyrgops, Neopyrgops,

333 Alcidodes) and long-horned beetles (e.g. Doliops, Paradoliops) mimic Pachyrhynchus'

334 aposematic signals to ward off predators. Currently, the Pachyrynchini has 17 known genera,

335 with the majority found exclusively in the Philippines (Schultze, 1923; Yap \& Gapud, 2007;

336 Yoshitake, 2013, 2018). 
Pachyrhynchus Germar, 1824 has the widest geographic range among Pachyrynchini.

338 There are presently 145 species in the genus, of which $93 \%$ of are endemic to the Philippines

339 (Rukmane, 2018), with the majority of species having a narrow geographic range, limited to a

340 mountain range, island, or Pleistocene Aggregate Island Complex (PAIC) (Inger 1954, Heaney

341 1985, Brown and Siler 2014). The general diagnostic characters of Pachyrhynchus Germar, 1824

342 include a head lacking a distinct transverse groove or distinct basal border, entire episternal

343 suture, and antennal scape not reaching the hind eye (Schultze, 1923; Yoshitake, 2012). $P$.

344 sulphureomaculatus Schultze, 1922, is only recorded from Mindanao Island (Schultze, 1922;

345 Cabras et al., 2017; Rukmane, 2018). This species was described from material collected in

346 South Cotabato but has recently been recorded (personal observations of A. Cabras) in other

347 areas of Mindanao (e.g. Marilog, Davao City, Arakan, Cotabato, Mt. Kiamo, Bukidnon). This

348 species belongs to the P. venustus group, conspicuous for their large size, prothorax with two

349 dorsolateral spots in the middle a large, oblong spot at the lateral margins, and elytra with oval or 350 oblong spots (Schultze, 1923).

352 Collection and extraction of DNA

353 Specimens were collected near the edge of the road in a secondary forest (HWY 81,

354 Arakan, Cotabato, Philippines [N7.487059, E125.248795]). One individual was used for both in

355 situ Hi-C and high molecular weight DNA libraries. A second individual was used for

356 transcriptome sequencing. Individuals were collected live, then frozen and stored at $-80^{\circ} \mathrm{C}$ until

357 library preparation.

358 Beetle tissues were dissected carefully to avoid inclusion of contaminants from guts and

359 impurities from chitinous cuticles. Half of the resulting tissues were used for Phenol Chloroform 
360 (PCI) based high molecular weight (HMW) DNA extraction for PacBio sequencing (the other

361 half of the material was used as starting material for Hi-C library preparation, see below).

362 Tissues were homogenized on ice using a sterile razor blade. ATL buffer $(140 \mu 1)$ and Proteinase

$363 \mathrm{~K}(60 \mu \mathrm{l})$ were then added to the homogenized material and incubated at $65^{\circ} \mathrm{C}$ for $1 \mathrm{hr}$. The 200

$364 \mu$ of resulting lysate was used as starting material for the PCI extraction following a PacBio

365 recommended protocol. (https://www.pacb.com/wp-content/uploads/2015/09/SharedProtocol-Extracting-DNA-usinig-Phenol-

366 Chloroform.pdf). Two additional rounds of PCI clean-up were performed to eliminate impurities such

367 as chitin to meet the DNA requirement for PacBio sequencing. In particular, to achieve OD

368 ratios of 1.8-2.0. DNA concentration was determined with the Qubit ${ }^{\mathrm{TM}}$ dsDNA HS Assay Kit

369 (Invitrogen corp., Carlsbad, CA), and high molecular weight content was confirmed by running a

370 Femto Pulse (Agilent, Santa Clara, USA).

\section{In situ Hi-C library preparation}

Tissues from the same sample were homogenized using a sterile razor blade on ice. An in

374 situ Hi-C library was prepared as described in Rao et al. (2014) with a few modifications.

375 Briefly, after the Streptavidin Pull-down step, the biotinylated Hi-C products underwent end

376 repair, ligation and enrichment using the NEBNext ${ }^{\circledR}$ Ultra ${ }^{\mathrm{TM}}$ II DNA Library Preparation kit

377 (New England Biolabs Inc, Ipswich, MA). Furthermore, titration of the number of PCR cycles

378 was performed as described in Belton et al. (2012).

\section{Transcriptome library preparation}

RNA extraction was performed using tissues from a frozen sample. Tissue was extracted

382 from the prothorax and abdomen with the digestive tract removed. The Monarch Total RNA 
383 Miniprep kit (New England Biolabs Inc, Ipswich, MA) was used for extraction. The

384 manufacturer's protocol for total RNA purification from tissue was followed (cite)

385 (https://www.neb.com/protocols/2017/11/08/total-rna-purification-from-tissues-and-leukocytes-using-the-monarch-total-rna-miniprep-kit-neb-

386 t2010). RNA concentration was determined using the Qubit ${ }^{2}$ RNA HS Assay Kit (Invitrogen

387 corp., Carlsbad, CA), and intact RNA content was confirmed by running a Bioanalyzer High

388 Sensitivity RNA Analysis (Agilent, Santa Clara, USA). The resulting RNA was sent to

389 Novogene Inc. for library preparation and sequencing, from which $12.5 \mathrm{Gbp}$ of data were

390 obtained.

\section{Genome sequencing and assembly}

First, we performed an initial quality control of the in situ Hi-C library using the CPU

394 version of Juicer v 1.5.7 (Durand et al. 2016) to determine if enough ligation motifs were present

395 in the sample. To accomplish this, we first cleaned our reads with fastp (Chen et al. 2018) to

396 remove sequencing adapters and low quality reads with default settings except for the more

397 sensitive '--detect_adapter_for_pe' setting on. After passing the quality control of having $>30 \%$

398 ligation motifs present, we proceeded to sequence the full library at higher coverage. We only

399 considered ligation motifs as this was a de novo assembly without a closely related reference

400 genome to align to the Hi-C reads. The full Hi-C library was sequenced on a paired-end $(2 \times 150$

401 bp) lane on an Illumina HiSeq4000. High molecular weight DNA was sent to the QB3 Genomics

402 facility at the University of California Berkeley for sequencing on a Pacific Biosciences Sequel

403 II platform, sequencing one cell with CLR version 2 chemistry (PacBio, Menlo Park, CA, USA).

$404 \quad$ We used PacBio Assembly Tool Suite pb-assembly v 0.0.8 (which includes the FALCON

405 assembly pipeline) to assemble the primary scaffolds. Next, we polished the primary assembly

406 using 3 rounds of mapping the raw fastq reads using minimap2 (Li 2018) followed by using 
RACON (Vaser 2017) to help error correct the initial assembly. This was followed by running the Purge_Haplotigs (Roach et al. 2018) pipeline to eliminate haplotigs (alternative haplotype contigs) in the assembly. Next, using the CPU version of Juicer v 1.5.7, we created a site

410 positions file for the restriction enzyme MboI using Juicer's generate_site_positions.py script,

411 followed by running Juicer until it creates the mapping stats file and a "merged_nodups" file.

412 Then we used the 3D-DNA (Dudchenko et al. 2017) pipeline with default settings to correct

413 misjoins and place scaffolds into chromosome groups. After generating a Hi-C heat map, we

414 corrected any assembly errors manually via Juicebox Assembly Tools v 1.11.08 (Durand et al.

415 2016, Dudchenko et al. 2018). After, (Fig 1.) we ran 3D-DNA's run-asm-pipeline-post-review.sh

416 to produce a final assembly file and fasta. To polish our final assembly further, we aligned our

417 Hi-C reads to our scaffolds using bwa mem followed by SAMclip and SAMtools 'view' (Li et al.

418 2009) with options '-S -b -f 2 -q 1 -F 1536'. After grouping scaffolds into chromosomes, we

419 divided each into a separate fasta (due to memory constraints) and used Pilon (v. 1.23) (Walker

420 et al. 2014) in "--fix bases" mode as to not break our scaffolds and to fix any homopolymer

421 repeat errors. The resulting assembly was used in all subsequent analyses.

\section{Removal of mitochondrial/contaminant DNA}

To identify scaffolds that contained mitochondrial cytochrome oxidase subunit 1 (COI) DNA, we used BLAT v. 35 (Kent 2002; 2012) using a reference sequence from Pachyrhynchus

426 smaragdinus (Supplemental Information file "P79_coI.fasta") to query our scaffolds. Once

427 identified, these scaffolds were removed. We also used blast (Camacho et al. 2008) with the nt

428 database and default settings to identify contaminant (non-arthropod or undetermined) sequences

429 and then removed these from the final assembly. These represented only a handful of sequences. 
Repeat content analyses

To address what is making the genome of Pachyrhynchus sulphureomaculatus so large

433 relative to other complete weevil genomes (>85\% Benchmarking Universal Single-Copy

434 Orthologs BUSCO Insecta genes), we compared the repeat content of P. sulphureomaculatus to

4355 other weevil genomes from NCBI (Supplemental Information file

436 “NCBI_numbers_for_Weevils_used_in_repeatmasker”). We used the de novo RepeatModeler v.

437 open-1.0.11 (Smit et al. 2015) repeat set combined with all repbase recs to first model for repeat

438 content. Next, we used RepeatMasker v. 4.1.0 (Smit et al. 2015) to annotate and soft mask repeat

439 content. For Listronotus, we downloaded the results from Harrop et al. (2020), who used

440 comparable methodologies. We also calculated the percentage of repetitive content (bases soft

441 masked) in a $1 \mathrm{Mb}$ sliding window across the chromosomes in $R$ using a custom script.

Genome annotation

We first cleaned our reads with fastp and concatenated the unpaired cleaned reads. We

445 performed 3 different initial reconstructions of the transcriptome: 1) Trinity v. 2.11.0 (Grabherr

446 et al. 2013; Haas et al. 2014) de novo assembly using default settings, 2) Trinity genome guided

447 assembly, where we first aligned our reads with tophat v. 2.1.1 (Kim et al. 2013),

448 3) rnaSPAdes (Bushmanova et al. 2019) de novo assembly. Selecting the rnaSPAdes assembly,

449 because it had the most single copy BUSCO V2 Arthropoda genes (Felipe et al. 2015), we

450 mapped our reads to this soft masked assembly using HISAT2 v. 2.2.0 (Kim et al. 2019), and

451 formatted a bam file using SAMtools 'view -b -f 3 -F 256 -q 10'. Next, we used BRAKER v. 2.1.5

452 (Hoff et al. 2019) to create an annotated gff. This process used the bam file from HISAT2 and 
453 results from a BUSCO search as 'seeding' genes to make the resulting gff. In addition, we used

454 the PASA pipeline (Campbell et al. 2006; Haas 2008) which used our rnaSPAdes transcripts

455 aligned to the genome assembly with BLAT (Kent 2002) and gmap (Wu and Watanabe 2005).

456 Lastly, we used EVidenceModeler (Haas et al. 2008) to evaluate our different annotations using

457 the developers' recommended weights for each assembly type to produce the final gene model

458 gff.

Synteny across coleopteran and Insecta chromosome-level genomes

461 To examine the gene synteny between other Coleoptera genomes, we downloaded chromosome-

462 level genomes from NCBI or supplied form the journal or authors website (Supplemental

463 Information file "NCBI_all_taxa_genomes_list") (Fallon et al. 2018; Zhang et al. 2020; Herndon

464 et al. 2020; Van Belleghem 2018). We also used the unpublished genome assemblies (Tribolium

465 castaneum [GCF_000002335.3], Bombyx mori [GCA_000151625.1], Clogmia albipunctata

466 [clogmia.6], Culex quinquefasciatus [CpipJ3], and Rhodnius prolixus [Rhodnius_prolixus-

$4673.0 .3]$ ), generated by the DNA Zoo Consortium (dnazoo.org). The assemblies were based on the

468 whole genome sequencing data from (Herndon et al., 2020; Tribolium Genome Sequencing

469 Consortium 2008, International Silkworm Genome Consortium 2008, Arensburger et al. 2010,

470 Mesquita et al. 2015) as well as Hi-C data generated by the DNA Zoo Consortium and

471 assembled using 3D-DNA (Dudchenko et al., 2017) and Juicebox Assembly Tools

472 (Dudchenko et al., 2018). Next, we identified the BUSCO v.2 loci, (1658 Insecta gene set) and 473 extracted their coordinates for the single and fragmented loci. We then compared the coordinates

474 of Pachyrhynchus sulphureomaculatus to the other Coleoptera genomes. Following, we

475 calculated the number of loci found in P. sulphureomaculatus chromosomes and those in the 
476 other Coleoptera and calculated the percent conserved within a chromosome. To visualize the

477 shared synteny, we plotted the different pairs using the R package RIdeogram (Hao et al. 2020).

478 To help visualize the relationship between the different taxa, we generated an ultraconserved

479 elements (UCE) dataset between the taxa using the PHYLUCE pipeline (Faircloth 2012). We

480 used the loci to help reconstruct a concatenated phylogeny in RAxML (Stamatakis 2014) and

481 calculate branch lengths to render the tree ultrametric. We dated the tree using dates (95\%

482 highest posterior density interval HPD) from McKenna et al. (2019) using the R package ape

483 v.5.4 'makeChronosCalib’ function (Paradis and Schliep 2019) (see Supplemental Information

484 file "Insecta_Claibrations_table" for dates).

Next, we investigated whether the observed synteny was distinctive within Coleoptera

486 relative to other orders of insects, such as Lepidoptera, in which high levels of synteny between

487 taxa have been recorded (Hill et al. 2019, Ahola et al. 2014). We used all insect genomes (with

488 some exceptions) available from NCBI that were marked as "chromosome" level. (See

489 Supporting Information for a complete list.) We tried to sample evenly across insect orders. For

490 example, we excluded the many Drosophila genomes as they are all phylogenetically close

491 relatives, and this would cause over-representation (i.e. we want patterns of chromosomal

492 evolution across Diptera, not just Drosophila). Instead, we sampled individual species across the

493 phylogenetic breadth of the genus. In addition, we also gathered genomes from the literature.

494 (see Supplemental Information file "NCBI_all_taxa_genomes_list”.) Next, we identified all

495 BUSCO version 5-beta loci that were single copy and calculated the gene order conservation

496 (GOC) score (see https://m.ensembl.org/) using a custom script (Supplemental Information files

497 “1make_scaff_order_busco_tsv.sh", and "2busco_GOC.sh"). First, we ordered the BUSCO v5-

498 beta genes by scaffold and position and then identified two genes upstream and downstream 
499 from a particular gene. Next, to determine if a set of 4 genes are in the same order in our target

500 genome, they receive a score of $1,0.75,0.5,0.25$ or 0 based on whether $4,3,2,1$ or 0 genes are

501 in the same order, respectively. Missing genes between the two genomes are discarded from

502 comparisons. This process is repeated along the length of the two genomes. We then summed the

503 scores for the four categories $0-100 \%$ and added these categories together (e.g. if 8 matched sets

504 were found at $25 \%$ and 1 at $100 \%$, the total score would be 5). These total scores were

505 normalized by dividing by the minimum number of genes present in the comparisons. We

506 computed the total GOC scores for all pairwise comparisons among the 34 taxa. Next, to

507 consider the effect of the phylogenetic relationships, we reconstructed the relationship among

508 our taxa using the BUSCO gene sets' amino acids. We used custom scripts to identify a 70\%

509 complete matrix and used mafft with 1000 iterations and the "localpair" settings to align the

510 sequences. Next, we used trimAI (Capella-Gutierrez et al. 2009) with "automated1" settings to

511 remove ambiguously aligned positions. RAxML-ng with the $\mathrm{LG}+\mathrm{G} 8+\mathrm{F}$ site rate substitution

512 model was used to reconstruct the phylogeny for our exemplar taxa across Insecta. We calibrated

513 our tree using the same methods for the beetle tree (above), and calibration points can be found

514 in the Supplemental Information file "Insecta_Claibrations_table”, from Misof et al 2014,

515 Obbard et al. 2012 and Mckenna et al. 2019). This calibration was done to help visualize the data

516 as the subsequent Mantel test did not require an ultrametric tree. Lastly, to test if pairwise

517 phylogenetic distance covaries with pairwise synteny values, we conducted a Mantel test.

\section{Data availability}

520 Assembly available at https://www.dnazoo.org/

521 Supplemental Information available on Dryad [link pending submission] 
522 Sequencing data from this project are archived on the SRA under accession \#\#\#\#\#\#

523 [embargoed until 2021-11-01 or publication].

525 Acknowledgments

526 We would like to thank the Ruth Tawan-tawan, Ceso II of the Philippines' Department of

527 Environment and Natural Resources Region XI for help with the Gratuitous and export permits.

528 We would also like to thank the University of Mindanao for the mobility support, and Milton N.

529 Medina and Chrestine Torrejos of U.M. for help collecting specimens. We would like to thank

530 Zane Colaric of B.C.M., for the help loading the QC library runs. We would also like to thank

531 Sarah Crews of C.A.S. for help with the manuscript text. We were funded in part through

532 NSF:DEB award number 1856402 made to MHVD.

$534 \quad$ Figure Legends

535

536 Figure 1. Pachyrhynchus sulphureomaculatus, lateral habitus. (Photo by A. Cabras)

538 Figure 2. Hi-C contact map heatmap of Pachyrhynchus sulphureomaculatus Schultze, 1922.

539 Eleven chromosome boundaries are indicated by black lines. Heatmap scale lower left, range in

540 counts of mapped Hi-C reads per megabase squared. Rabl-like pattern highlighted along

541 chromosome 1, top row, open triangles indicate contact between centromere regions. X-like

542 pattern between adjacent off diagonal regions indicative of contact between distal portions of

543 chromosomes. 
545 Figure 3. P. sulphureomaculatus scaffold bubble plot of coverage versus GC content. Scaffolds

546 included are from the unfiltered assembly. Taxonomic annotation provided via blastn alignment

547 to the NCBI nt database.

549 Figure 4. Stacked bar plot of Insecta BUSCO gene sets by category for chromosome-level beetle

550 genomes. Y-axis is the percent of BUSCO genes, $\mathrm{X}$-axis labels are the genus names. The

551 abbreviations in the legend are: $\mathrm{D}=$ duplicated, $\mathrm{F}=$ fragmented, $\mathrm{M}=$ missing and $\mathrm{S}=$ single.

553 Figure 5. Histogram of repeat content for weevil genomes.

555 Figure 6. Heat map of gene density and non-repetitive DNA per $1 \mathrm{Mb}$ sliding window. The 11

556 chromosomes are in the same order as in the Hi-C heat map (Fig. 1) and fasta file.

Figure 7. Chronogram and ideograms of the 5 beetle genomes which have chromosome level

559 assemblies.

Figure 8. Stacked bar plots and chromosome mappings of BUSCO genes' placements. Colors

562 correspond to $P$. sulphureomaculatus chromosomes. The numbering scheme of chromosomes

563 matches the names found in the genome's fasta file.

565 Figure 9. Insecta, gene order conservation score (GOC) plot. Left panel, chronogram, branches

566 colored by order. Heat map of normalized pairwise GOC scores, redder boxes indicate more 

phylogenetic distance.

569

570

571

572

573

574

581

582

\begin{tabular}{|l|r|}
\hline Number of scaffolds & 4,093 \\
\hline Total size of scaffolds & $2,051,389,195$ \\
\hline Number of contigs & 14,365 \\
\hline Number of contigs in scaffolds & 10,283 \\
\hline Number of contigs not in scaffolds & 4,082 \\
\hline Mean scaffold size & 501,195 \\
\hline Median scaffold size & 8,175 \\
\hline N50 scaffold length & $215,921,627$ \\
\hline scaffold \%AT & 33.11 \\
\hline scaffold \%CG & 16.87 \\
\hline scaffold \%N & 0.05 \\
\hline \% bp of assembly in chromosomes & $\mathbf{9 7 . 5 2}$ \\
\hline
\end{tabular}

\section{TABLES}

Inter-chromosomal: 56,711,177 (24.85\%/38.63\%)

Intra-chromosomal: $23,941,704(10.49 \% / 16.31 \%)$

Short Range (<20Kb): 18,227,329 (7.99\% / 12.42\%)

Long Range (>20Kb): 5,714,353 (2.50\%/ 3.89\%)

Table 1. Summary of Hi-C reads mapped.

Table 2. Summary statistics for final assembly. 


\begin{tabular}{|lllllll|}
\hline Chromosome & length bp & $\begin{array}{c}\text { \# of } \\
\text { contigs }\end{array}$ & $\begin{array}{c}\text { number of } \\
\text { N's (runs of }\end{array}$ & $\begin{array}{c}\text { percent N's } \\
\text { in chromo. }\end{array}$ & N50 & $\begin{array}{c}\text { N50 reached in } \\
\text { \# of contigs }\end{array}$ \\
\hline Chr_1 & $263,832,947$ & 1388 & 138,700 & $0.05 \%$ & 287319 & 280 \\
Chr_2 & $253,284,860$ & 1222 & 122,100 & $0.04 \%$ & 326447 & 246 \\
Chr_3 & $137,890,936$ & 683 & 68,200 & $0.04 \%$ & 315991 & 127 \\
Chr_4 & $223,502,247$ & 1131 & 113,000 & $0.05 \%$ & 297265 & 217 \\
Chr_5 & $69,931,891$ & 236 & 23,500 & $0.03 \%$ & 452330 & 50 \\
Chr_6 & $125,299,487$ & 655 & 65,400 & $0.05 \%$ & 304080 & 131 \\
Chr_7 & $132,078,125$ & 624 & 62,300 & $0.04 \%$ & 368684 & 112 \\
Chr_8 & $173,282,956$ & 836 & 83,500 & $0.04 \%$ & 335626 & 165 \\
Chr_9 & $215,921,627$ & 1221 & 122,000 & $0.05 \%$ & 280522 & 237 \\
Chr_10 & $186,927,849$ & 988 & 98,700 & $0.05 \%$ & 290475 & 197 \\
Chr_11 & $218,628,933$ & 1074 & 107,300 & $0.04 \%$ & 316887 & 219 \\
\hline
\end{tabular}

Table 3. Summary statistics for final assembly by chromosome.

\section{REFERENCES}

Ahola V, Lehtonen R, Somervuo P, Salmela L, Koskinen P, Rastas P, Välimäki N, Paulin L, Kvist J, Wahlberg N, et al. 2014. The Glanville fritillary genome retains an ancient karyotype and reveals selective chromosomal fusions in Lepidoptera. Nature Communications 2014;5 doi: 10.1038/ncomms5737. Article 4737 
Anbutsu H, Moriyama M, Nikoh N, Hosokawa T, Futahashi R, Tanahashi M, Meng XY, Kuriwada T, Mori N, Oshima K, Hattori M. 2017. Small genome symbiont underlies cuticle hardness in beetles. Proceedings of the National Academy of Sciences 114:E8382-E8391.

Arensburger P, Megy K, Waterhouse RM, Abrudan J, Amedeo P, Antelo B, Bartholomay L, Bidwell S, Caler E, Camara F, et al. 2010. Sequencing of Culex quinquefasciatus establishes a platform for mosquito comparative genomics. Science. 330(6000):86-8. doi:10.1126/science.1191864.

Belton JM, McCord RP, Gibcus JH, Naumova N, Zhan Y, Dekker J. 2012. Hi-C: a comprehensive technique to capture the conformation of genomes. Methods 58:268-276.

Biello R, Singh A, Godfrey CJ, Fernández FF, Mugford ST, Powell G, Hogenhout SA, Mathers TC. 2020. A chromosome level genome assembly of the woolly apple aphid, Eriosoma lanigerum Hausmann (Hemiptera: Aphididae). Molecular Ecology Resources 00: 1-11. https://doi.org/10.1111/1755-0998.13258

Bracewell R, Chatla K, Nalley MJ, Bachtrog D. 2019. Dynamic turnover of centromeres drives karyotype evolution in Drosophila. eLife 2019;8:e49002.

Brown RM and Siler CD. 2014. Spotted stream frog diversification at the Australasian faunal zone interface, mainland versus island comparisons, and a test of the Philippine 'dual-umbilicus' hypothesis. Journal of Biogeography. 41:182-195.

Bushmanova E, Antipov D, Lapidus A, Prjibelski AD. 2019. rnaSPAdes: a de novo transcriptome assembler and its application to RNA-Seq data. GigaScience. 8:giz100, https://doi.org/10.1093/gigascience/giz100

Campbell MA, Haas BJ, Hamilton JP, Mount SM, Buell CR. 2006. Comprehensive analysis of alternative splicing in rice and comparative analyses with Arabidopsis. BMC Genomics 2006, 7:327 http://www.biomedcentral.com/1471-2164/7/327

Chen S, Zhou Y, Chen Y, Gu J. 2018. fastp: an ultra-fast all-in-one FASTQ preprocessor. Bioinformatics 34:884-890. doi: 10.1093/bioinformatics/bty560

Capella-Gutierrez S, Silla-Martinez JM, Gabaldon T. 2009. trimAl: a tool for automated alignment trimming in large-scale phylogenetic analyses. Bioinformatics 25:19721973.

Camacho C, Coulouris G, Avagyan V, Ma N, Papadopoulos J, Bealer K, Madden TL. 2008. BLAST+: architecture and applications. BMC Bioinformatics 10:421.

Cabras A, Coritico F, Mohagan A, Rukname A. 2017. Pachyrynchini of Mt. Kiamo, Malaybalay, Bukidnon, Mindanao Island. Journal of Entomology and Zoology Studies. 5(3):979-983. 
Csink AK and Henikoff S. 1998. Large-scale chromosomal movements during interphase progression in Drosophila. J. Cell Biol. 143:13-22.

Chowdhary BP, Raudsepp T, Frönicke L, Scherthan H. 1998. Emerging Patterns of Comparative Genome Organization in Some Mammalian Species as Revealed by Zoo-FISH. Genome Res. 8:577-589. doi:10.1101/gr.8.6.577

Ceja-Navarro JA, Karaoz U, Bill M, Hao Z, White RA 3rd, Arellano A, Ramanculova L, Filley TR, Berry TD, Conrad ME, et al. 2019. Gut anatomical development and microbial functional assembly promote lignocellulose deconstruction and colony subsistence of a wood-feeding beetle. Nat. Microbiol. 4:864-75.

Concia L, Veluchamy A, Ramirez-Prado JS, Martin-Ramirez A, Huang Y, Perez M, Domenichini S, Rodriguez Granados NY, Kim S, Blein T, Duncan S, et al. Wheat chromatin architecture is organized in genome territories and transcription factories. Genome Biol. 21(1):104. doi: 10.1186/s13059-020-01998-1.

Cowan CR, Carlton PM, Cande WZ. 2001. The Polar Arrangement of Telomeres in Interphase and Meiosis. Rabl Organization and the Bouquet. Plant Physiology 125(2):532-538. DOI: $10.1104 / \mathrm{pp} .125 .2 .532$

Davey JW, Chouteau M, Barker SL, Maroja L, Baxter SW, Simpson F, Merrill RM, Joron M, Mallet J, Dasmahapatra KK, Jiggins CD. 2016. Major Improvements to the Heliconius melpomene Genome Assembly Used to Confirm 10 Chromosome Fusion Events in 6 Million Years of Butterfly Evolution. G3 (Bethesda) 6(3):695708.

Deakin JE. 2018. Chromosome Evolution in Marsupials. Genes. 9: 72.

Dudchenko O, Batra SS, Omer AD, Nyquist SK, Hoeger M, Durand NC, Shamim MS, Machol I, Lander ES, Aiden AP, Aiden EL. 2017. De novo assembly of the Aedes aegypti genome using Hi-C yields chromosome-length scaffolds. Science 356:9295. https://doi.org/10.1126/science.aal3327.

Dudchenko O, Shamim MS, Batra S, Durand NC, Musial NT, Mostofa R, Pham M, et al. 2018. The Juicebox Assembly Tools Module Facilitates de Novo Assembly of Mammalian Genomes with Chromosome-Length Scaffolds for under \$1000. bioRxiv, January, 254797. https://doi.org/10.1101/254797

Durand NC, Shamim MS, Machol I, Rao SS, Huntley MH, Lander ES, Aiden EL. 2016. Juicer Provides a One-Click System for Analyzing Loop-Resolution Hi-C Experiments. Cell Syst. 3(1):95-8. doi:10.1016/j.cels.2016.07.002.

Eagen KP, Aiden EL, Kornberg RD. 2017. Polycomb-mediated chromatin loops revealed by a subkilobase-resolution chromatin interaction map. Proc. Natl. Acad. Sci. 114:8764-8769. 
Fallon TR, Lower SE, Chang CH, Bessho-Uehara M, Martin GJ, Bewick AJ, Behringer M, Debat HJ, Wong I, Day JC, et al. 2018. Firefly genomes illuminate parallel origins of bioluminescence in beetles. eLife. 2018;7:e36495. pmid:30324905

Ghurye J, Pop M, Koren S, Bickhart D, Chin CS. 2017. Scaffolding of long-read assemblies using long range contact information. BMC genomics. 18(1):527.

Ghurye J, Rhie A, Walenz BP, Schmitt A, Selvaraj S, Pop M, Phillippy AM, Koren S. Integrating Hi-C links with assembly graphs for chromosome-scale assembly. 2019. PLoS Comput Biol. 15(8):e1007273. doi:10.1371/journal.pcbi.1007273.

Grabherr MG, Haas BJ, Yassour M, Levin JZ, Thompson DA, Amit I, Adiconis X, Fan L, Raychowdhury R, Zeng Q, et al. 2011. Full-length transcriptome assembly from RNA-Seq data without a reference genome. Nat Biotechnol. 29(7):644-52. doi: 10.1038/nbt.1883.

Goto B, Okazaki K, Niwa O. 2001. Cytoplasmic microtubular system implicated in de novo formation of a Rabl-like orientation of chromosomes in fission yeast. $J$ Cell Sci. 114:2427-35.

Haas BJ, Papanicolaou A, Yassour M, Grabherr M, Philip D, Bowden J, Couger MB, Eccles D, Li B, Macmanes MD, Ott M, Orvis J, Pochet N: Reference Generation and Analysis with Trinity. Volume 8; 2014.

Haas BJ. 2008. Analysis of Alternative Splicing in Plants with Bioinformatics Tools. Nuclear pre-mRNA Processing in Plants.

Haas BJ, Salzberg SL, Zhu W, Pertea M, Allen JE, Orvis J, White O, Buell CR, Wortman JR. 2008. Automated eukaryotic gene structure annotation using EVidenceModeler and the Program to Assemble Spliced Alignments. Genome Biol. 9(1):R7. doi: 10.1186/gb-2008-9-1-r7.

Hammond PM. 1992. Species inventory. Global Biodiversity. Status of the Earth's Living Resources. A Report Compiled by the World Conservation Monitoring Centre, ed Groombridge B (Chapman and Hall, London), pp 17-39.

Hao Z, Lv D, Ge Y, Shi J, Weijers D, Yu G, Chen J. 2020. RIdeogram: drawing SVG graphics to visualize and map genome-wide data on the idiograms. PeerJ Comput. Sci. 6:e251 http://doi.org/10.7717/peerj-cs.251

Harrop T, Le Lec MF, Jauregui R, Taylor SE, Inwood SN, van Stijn T, Henry H, Skelly J, Ganesh S, Ashby RL, Jacobs J, Goldson SL, Dearden PK. 2020. Genetic Diversity in Invasive Populations of Argentine Stem Weevil Associated with Adaptation to Biocontrol. Insects, 11(7), 441. https://doi.org/10.3390/insects11070441 
Heaney LR, 1985. Zoogeographic evidence for middle and late pleistocene land bridges to the philippine islands. Mod Quatern Res SE Asia 9:127-144.

Hill J, Rastas P, Hornett EA, Neethiraj R, Clark N, Morehouse N, de la Paz CelorioMancera M, Cols JC, Dircksen H, Meslin C, et al., 2019. Unprecedented reorganization of holocentric chromosomes provides insights into the enigma of lepidopteran chromosome evolution. Sci. $A d v$. 5:1-13.

Edelman NB, Frandsen PB, Miyagi M, Clavijo B, Davey J, Dikow R, García-Accinelli G, Van Belleghem SM, Patterson N, Neafsey DE, et al. Genomic architecture and introgression shape a butterfly radiation. 2019. Science. 366(6465):594-599. doi: 10.1126/science.aaw2090

Herndon N, Shelton J, Gerischer L, Ioannidis P, Ninova M, Dönitz J, Waterhouse RM, Liang C, Damm C, Siemanowski J, et al. 2020. Enhanced genome assembly and a new official gene set for Tribolium castaneum. BMC Genomics. 21(1):47. doi: 10.1186/s12864-019-6394-6.

Hoff KJ, Lomsadze A, Borodovsky M, Stanke M. 2019. Whole-Genome Annotation with BRAKER. Methods Mol Biol. 1962:65-95. doi:10.1007/978-1-4939-9173-0_5

Hsu CF, Tseng HY, Hsiao Y, Ko CC. 2017. First record of the host plant and larvae of Pachyrhynchus sonani (Coleoptera: Curculionidae) on Lanyu Island, Taiwan. Entomological Science, 20:288-291. doi: 10.1111/ens.12262.

Idziak D, Robaszkiewicz E, Hasterok R. 2015. Spatial distribution of centromeres and telomeres at interphase varies among Brachypodium species. Journal of Experimental Botany. 66(21): 6623-6634. https://doi.org/10.1093/jxb/erv369

Inger RF, 1954. Systematics and zoogeography of Philippine Amphibia. Fieldiana 33:182-531.

Jin Q, Trelles-Sticken E, Scherthan H, Loidl J. 1998. Yeast nuclei display prominent centromere clustering that is reduced in nondividing cells and in meiotic prophase. The Journal of Cell Biology 141:21-29.

Kingan SB, Urban J, Lambert CC, Baybayan P, Childers AK, Coates B, Scheffler B, Hackett K, Korlach J, Geib SM. 2019. A high-quality genome assembly from a single, field-collected spotted lanternfly (Lycorma delicatula) using the PacBio Sequel II system. GigaScience, 8(10): giz122, https://doi.org/10.1093/gigascience/giz122

Kemkemer C, Kohn M, Cooper DN, Froenicke L, Högel J, Hameister H, KehrerSawatzki H. 2009. Gene synteny comparisons between different vertebrates provide new insights into breakage and fusion events during mammalian 
karyotype evolution. BMC evolutionary biology, 9, 84 .

https://doi.org/10.1186/1471-2148-9-84

Kent WJ. 2002. BLAT-The BLAST-Like Alignment Tool. Genome Research 12:656664. DOI: $10.1101 /$ gr.229202.

Kent WJ. 2012. BLAT-The BLAST-Like Alignment Tool. Version 35. Available at https://users.soe.ucsc.edu/ kent.

Kim D, Pertea G, Trapnell C, Pimentel H, Kelley R, Salzberg SL. 2013. TopHat2: accurate alignment of transcriptomes in the presence of insertions, deletions and gene fusions. Genome Biology 14:R36 (2013). https://doi.org/10.1186/gb-201314-4-r36.

Kim D, Paggi JM, Park C, Bennett C, Salzberg SL. 2019. Graph-based genome alignment and genotyping with HISAT2 and HISAT-genotype. Nat Biotechnol. 37(8):907-915. doi: 10.1038/s41587-019-0201-4.

Kim S, Liachko I, Brickner DG, Cook K, Noble WS, Brickner JH, Shendure J, Dunham MJ. 2017. The dynamic three-dimensional organization of the diploid yeast genome. eLife 2017;6:e23623 DOI: 10.7554/eLife.23623

Kingan SB, Heaton H, Cudini J, Holroyd N, Tracey A, Lambert CC, Baybayan P, Galvin B, Korlach J, Berriman M, Lawniczak, MKN. 2019. A Low DNA Input Protocol for High-quality PacBio De Novo Genome Assemblies from Single Invertebrate Individuals. https://www.pacb.com/proceedings/a-low-dna-input-protocol-forhigh-quality-pacbio-de-novo-genome-assemblies-from-single-invertebrateindividuals/

Li H. 2018. Minimap2: pairwise alignment for nucleotide sequences, Bioinformatics, 34(18):3094-3100. https://doi.org/10.1093/bioinformatics/bty191

Liao Y, Zhang X, Chakraborty M, Emerson JJ. 2020. Topologically associating domains and their role in the evolution of genome structure and function in Drosophila bioRxiv 2020.05.13.094516; doi: https://doi.org/10.1101/2020.05.13.094516

Lieberman-Aiden E, van Berkum NL, Williams L, Imakaev M, Ragoczy T, Telling A, Amit I, Lajoie BR, Sabo PJ, Dorschner MO, Sandstrom R, et al. 2009. Comprehensive mapping of long-range interactions reveals folding principles of the human genome. Science 326:289-293.

Liu Q, Guo Y, Zhang Y, Hu W, Li Y, Zhu D, Zhou Z, Wu J, Chen N, Zhou X-N. 2019. A chromosomal-level genome assembly for the insect vector for Chagas disease, Triatoma rubrofasciata. GigaScience, 8(8): giz089 https://doi.org/10.1093/gigascience/giz089 
Lu S, Yang J, Dai X, Xie F, He J, Dong Z, Mao J, Liu G, Chang Z, Zhao R, Wan W, Zhang R, Li Y, Wang W, Li X. 2019. Chromosomal-level reference genome of Chinese peacock butterfly (Papilio bianor) based on third-generation DNA sequencing and Hi-C analysis. GigaScience, 8(11):giz128 https://oi.org/10.1093/gigascience/giz128

Lukyanchikova V, Nuriddinov M, Belokopytova P, Liang J, Reijnders MJMF, Ruzzante L, Waterhouse RM, Tu Z, Sharakhov IV, Fishman V. 2020. Anopheles mosquitoes revealed new principles of 3D genome organization in insects. bioRxiv 2020.05.26.114017; doi: https://doi.org/10.1101/2020.05.26.114017

Lukhtanov VA, Dincă V, Friberg M, Šíchová J, Olofsson M, Vila R, Marec F, Wiklund C. 2018. Versatility of multivalent orientation, inverted meiosis, and rescued fitness in holocentric chromosomal hybrids. Proc Natl Acad Sci. 115(41):E9610E9619.

Mascher M, Gundlach H, Himmelbach A, Beier S, Twardziok SO, Wicker T, Radchuk V, Dockter C, Hedley PE, Russell J, Bayer M, et al. 2017. A chromosome conformation capture ordered sequence of the barley genome. Nature 544(7651):427-433. https://doi.org/10.1038/nature22043.

Marec F, Tothova A, Sahara K, Traut W. 2001. Meiotic pairing of sex chromosome fragments and its relation to atypical transmission of a sex-linked marker in Ephestia kuehniella (Insecta: Lepidoptera). Heredity. 87:659-671.

McKenna D.D., Sequeira A.S., Marvaldi A.E., Farrell B.D. 2009. Temporal lags and overlap in the diversification of weevils and flowering plants. Proceedings of the National Academy of Sciences. 106:7083-7088.

McKenna DD, Shin S, Ahrens D, Balke M, Beza-Beza C, Clarke DJ, Donath A, Escalona HE, Friedrich F, Letsch H, et al. 2019. The evolution and genome basis of beetle diversity. Proceedings of the National Academy of Sciences 116(49):2472924737.

Mathers TC, Wouters RHM, Mugford ST, Swarbreck D, van Oosterhout C, Hogenhout SA. 2020. Chromosome-scale genome assemblies of aphids reveal extensively rearranged autosomes and long-term conservation of the $\mathrm{X}$ chromosome. BioRxiv. doi: 10.1101/2020.03.24.006411

Matthews BJ, Dudchenko O, Kingan SB, Koren S, Antoshechkin I, Crawford JE, Glassford WJ, Herre M, Redmond SN, Rose NH, et al. 2018. Improved reference genome of Aedes aegypti informs arbovirus vector control. Nature. 563(7732):501-507. doi: 10.1038/s41586-018-0692-z.

Mesquita RD, Vionette-Amaral RJ, Lowenberger C, Rivera-Pomar R, Monteiro FA, Minx P, Spieth J, Carvalho AB, Panzera F, Lawson D, et al. 2015. Genome of Rhodnius prolixus, 
an insect vector of Chagas disease, reveals unique adaptations to hematophagy and parasite infection. Proceedings of the National Academy of Sciences. 112(48):1493614941. DOI: 10.1073 /pnas. 1506226112

Misof B, Liu S, Meusemann K, Peters RS, Donath A, Mayer C, Frandsen PB, Ware J, Flouri T, Beutel RG, et al. 2014. Phylogenomics resolves the timing and pattern of insect evolution. Science. 346:763-767.

Mizuguchi T, Barrowman J, Grewal SIS. 2015. Chromosome domain architecture and dynamic organization of the fission yeast genome. FEBS Letters. 589, doi: 10.1016/j.febslet.2015.06.008

Oberprieler RG. 2010. A reclassification of the weevil subfamily Cyclominae (Coleoptera: Curculionidae). Zootaxa 2515:1-35.

Obbard DJ, Maclennan J, Kim K-W, Rambaut A, O’Grady PM, Jiggins FM. 2012. Estimating Divergence Dates and Substitution Rates in the Drosophila Phylogeny. Molecular Biology and Evolution, 29(11): 3459-3473. https://doi.org/10.1093/molbev/mss 150

Oberprieler RG, Marvaldi AE, Anderson RS. 2007. Weevils, Weevils, Weevils Everywhere. Zootaxa 1668: 491-520. In Zhang, Z.-Q. and Shear, W.A. (eds.) (2007) Linnaeus Tercentenary: Progress in Invertebrate Taxonomy. Zootaxa 1668:1-766.

Paradis E, Schliep K. 2019. ape 5.0: an environment for modern phylogenetics and evolutionary analyses in R. Bioinformatics 35:526-528.

Pouokam M, Cruz B, Burgess S, Segal MR, Vazquez M, Arsuaga J. 2019. The Rabl configuration limits topological entanglement of chromosomes in budding yeast. Scientific Reports 9:1-10. https://doi.org/10.1038/s41598-019-42967-4/

Rao SS, Huntley MH, Durand NC, Stamenova EK, Bochkov ID, Robinson JT, Sanborn AL, Machol I, Omer AD, Lander ES, Aiden EL. 2014. A 3D Map of the Human Genome at Kilobase Resolution Reveals Principles of Chromatin Looping. Cell 159:1665-1680.

Renschler G, Richard G, Valsecchi CIK, Toscano S, Arrigoni L, Ramírez F, Akhtar A. 2019. Hi-C guided assemblies reveal conserved regulatory topologies on $X$ and autosomes despite extensive genome shuffling. Genes Dev. 33(21-22):1591-1612. doi: $10.1101 / \mathrm{gad} .328971 .119$.

Rabl C. 1885. Ueber Zelltheilung Morphol Jahrbuch, 10:214-330

Roach MJ, Schmidt SA, Borneman AR. 2018. Purge Haplotigs: allelic contig reassignment for third-gen diploid genome assemblies. BMC Bioinformatics 19:460. https://doi.org/10.1186/s12859-018-2485-7 
916

Rukmane A. 2018. An annotated checklist of genus Pachyrhynchus (Coleoptera: Curculionidae: Pachyrhynchini). Acta Biol. Univ. Daugavp. 18 (1):63-68.

SAMclip. https://github.com/tseemann/samclip. Accessed 1 May 2019

Sanborn AL, Rao SSP, Huang S-C, Durand NC, Huntley MH, Jewett AI, Bochkov ID, Chinnappan D, Cutkosky A, Li J, Geeting KP, Gnirke A, Melnikov A, McKenna D, Stamenova EK, Lander ES, Aiden EL. 2015. Extrusion predicts 3D genome engineering results. Proc. Natl Acad. Sci. USA. 112(47):E6456-E6465. DOI: $10.1073 /$ pnas. 1518552112

Schneider C, Woehle C, Greve C, D’Haese CA, Wolf M, Janke A, Bálint M, Hüttel B. 2020. Biodiversity genomics of small metazoans: high quality de novo genomes from single specimens of field-collected and ethanol-preserved springtails. bioRxiv 2020.08.10.244541; doi: https://doi.org/10.1101/2020.08.10.244541

Schultze W. 1922. Beitrag zur coleopteren fauna der Philippinen. Philippine Journal of Science 21(6): 569-596.

Schultze W. 1923. A monograph of the Pachyrrhynchid group of the Brachyderinae, Curculionidae: Part I. Philippine Journal of Science 23: 609-673.

Seppey M, Ioannidis P, Emerson BC, Pitteloud C, Robinson-Rechavi M, Roux J, Escalona HE, McKenna DD, Misof B, Shin S, Zhou X, Waterhouse RM, Alvarez N. 2019. Genomic signatures accompanying the dietary shift to phytophagy in polyphagan beetles. Genome Biol 20, 98 https://doi.org/10.1186/s13059-019$\underline{1704-5}$

Sheffer MM, Hoppe A, Krehenwinkel H, Uhl G, Kuss AW, Jensen L, Jensen C, Gillespie RG, Hoff KJ, Prost S. 2020 Chromosome-level reference genome of the European wasp spider Argiope bruennichi: a resource for studies on range expansion and evolutionary adaptation bioRxiv 2020.05.21.103564; doi: https://doi.org/10.1101/2020.05.21.103564

Simão FA, Waterhouse RM, Ioannidis P, Kriventseva EV, Zdobnov EM. BUSCO: assessing genome assembly and annotation completeness with single-copy orthologs, Bioinformatics, 31(19):3210-3212. https://doi.org/10.1093/bioinformatics/btv351

Simison BW, Parham JF, Papenfuss TJ, Lam AW, Henderson JB. 2020. An Annotated Chromosome-Level Reference Genome of the Red-Eared Slider Turtle (Trachemys scripta elegans). Genome Biol Evol. 12(4):456-462. doi: 10.1093/gbe/evaa063.

Smit AFA, Hubley R, Green P. 2015. RepeatMasker Open-4.0. 2015 $<$ http://www.repeatmasker.org $>$. 
962

963

964

965

966

967

968

969

970

971

972

973

974

975

976

977

978

979

980

981

982

983

984

985

986

987

988

989

990

991

992

993

994

995

996

997

998

999

1000

1001

1002

1003

1004

1005

1006

1007
Song C, Liu Y, Song A, Dong G, Zhao H, Sun W, Ramakrishnan S, Wang Y, Wang S, Li T, Niu Y, Jiang J, Dong B, Xia Y, Chen S, Hu Z, Chen F, Chen S. 2018. The Chrysanthemum nankingense genome provides insights into the evolution and diversification of chrysanthemum flowers and medicinal traits. Mol Plant. 11(12):1482-1491. doi:10.1016/j.molp.2018.10.003

Stamatakis A. 2014. RAxML version 8: a tool for phylogenetic analysis and post-analysis of large phylogenies, Bioinformatics, 30:(9)1312-1313.

https://doi.org/10.1093/bioinformatics/btu033

Stork NE, McBroom J, Gely C, Hamilton AJ. 2015. New approaches narrow global species estimates for beetles, insects and terrestrial arthropods. Proc. Natl Acad. Sci. USA 112, 7519-7523.

Tribolium Genome Sequencing Consortium, Richards S, Gibbs RA, Weinstock GM, Brown SJ, Denell R, Beeman RW, Gibbs R, Beeman RW, Brown SJ, et al. 2008. The genome of the model beetle and pest Tribolium castaneum. Nature. 452(7190):949-55. doi: 10.1038/nature06784.

Tseng H-Y, Lin C-P, Hsu J-Y, Pike DA, Huang W-S 2014. The Functional Significance of Aposematic Signals: Geographic Variation in the Responses of Widespread Lizard Predators to Colourful Invertebrate Prey. PLoS ONE 9(3):e91777. doi:10.1371/journal.pone.0091777

Vaser R, Sović I, Nagarajan N, Šikić M. 2017. Fast and accurate de novo genome assembly from long uncorrected reads. Genome Res. 27(5):737-746. doi:10.1101/gr.214270.116

Van Belleghem SM, Vangestel C, De Wolf K, De Corte Z, Möst M, Rastas P, De Meester L, Hendrickx F. 2018. Evolution at two time frames: Polymorphisms from an ancient singular divergence event fuel contemporary parallel evolution. PLoS Genet. 14(11):e1007796. doi: 10.1371/journal.pgen.1007796.

Wang W, Guan R, Liu X, Zhang H, Song B, Xu Q, Fan G, Chen W, Wu X, Liu X, Wang J. 2019. Chromosome level comparative analysis of Brassica genomes. Plant Mol Biol. 99(3):237-249. doi: 10.1007/s11103-018-0814-x.

Wu TD, Watanabe CK. 2005. GMAP: a genomic mapping and alignment program for mRNA and EST sequences. Bioinformatics. 21(9):1859-1875. https://doi.org/10.1093/bioinformatics/bti310

Yap SA, Gapud VP. 2007. Taxonomic review of the genus Metapocyrtus Heller (Coleoptera: Curculionidae: Entiminae: Pachyrrhynchini). Philip. Entomol. 21:115-135. 
Yoshitake H. 2013. A New Genus and Two New Species of the Tribe Pachyrhynchini (Coleoptera: Curculionidae) from Palawan Island, the Philippines. Esakia. 53:1-8.

Yoshitake H. 2018. A new genus and two new species of the tribe Pachyrhynchini (Coleoptera, Curculionidae, Entiminae) from the Philippines. Elytra 8(1):5-14.

Zhang L, Li S, Luo J, Du P, Wu L, Li Y, Zhu X, Wang L, Zhang S, Cui J. 2020. Chromosome-level genome assembly of the predator Propylea japonica to understand its tolerance to insecticides and high temperatures. Mol Ecol Resour. 20: 292- 307. https://doi.org/10.1111/1755-0998.13100

Zhou Y, Liang Y, Yan Q, Zhang L, Chen D, Ruan L, Kong Y, Shi H, Chen M, Chen J. 2020. The draft genome of horseshoe crab Tachypleus tridentatus reveals its evolutionary scenario and well developed innate immunity. BMC Genomics, 21(1):137-15. http://doi.org/10.1186/s12864-020-6488-1

Zimmerman EC. 1994. Australian weevils (Coleoptera: Curculionidae). Volume 1. Orthoceri: Anthribidae to Attelabidae: the primitive weevils. East Melbourne: CSIRO. pp. 741.

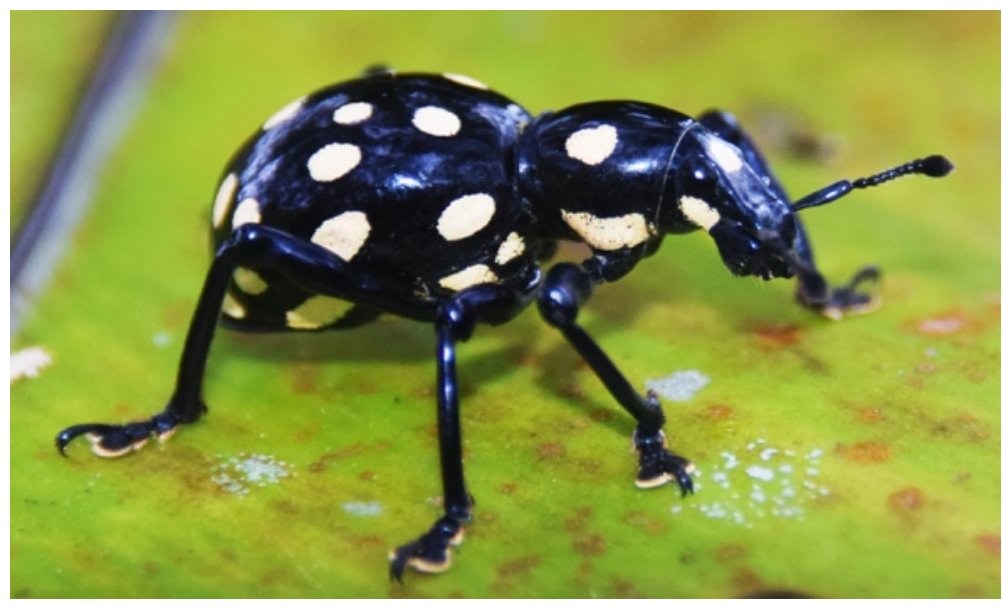




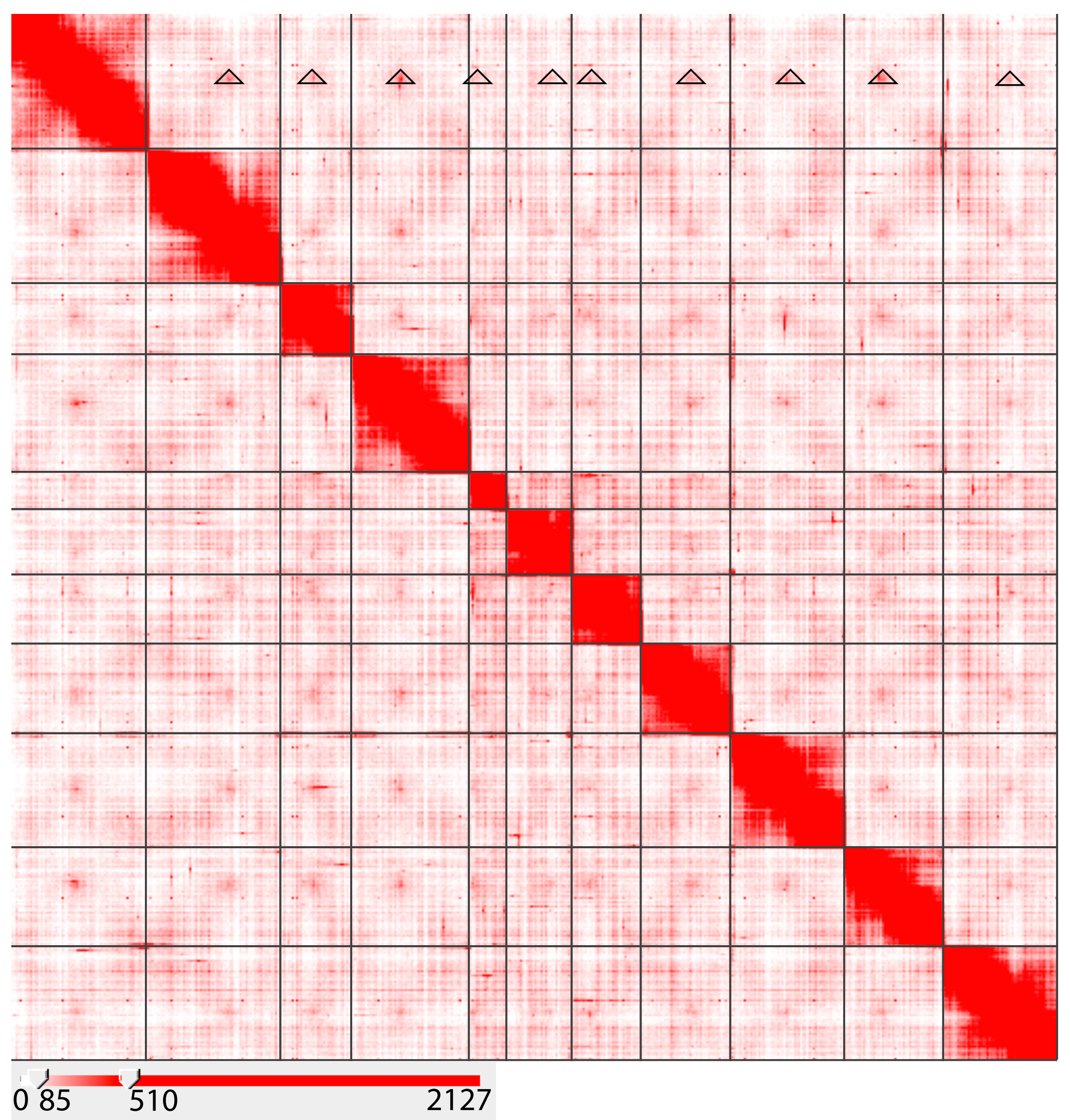




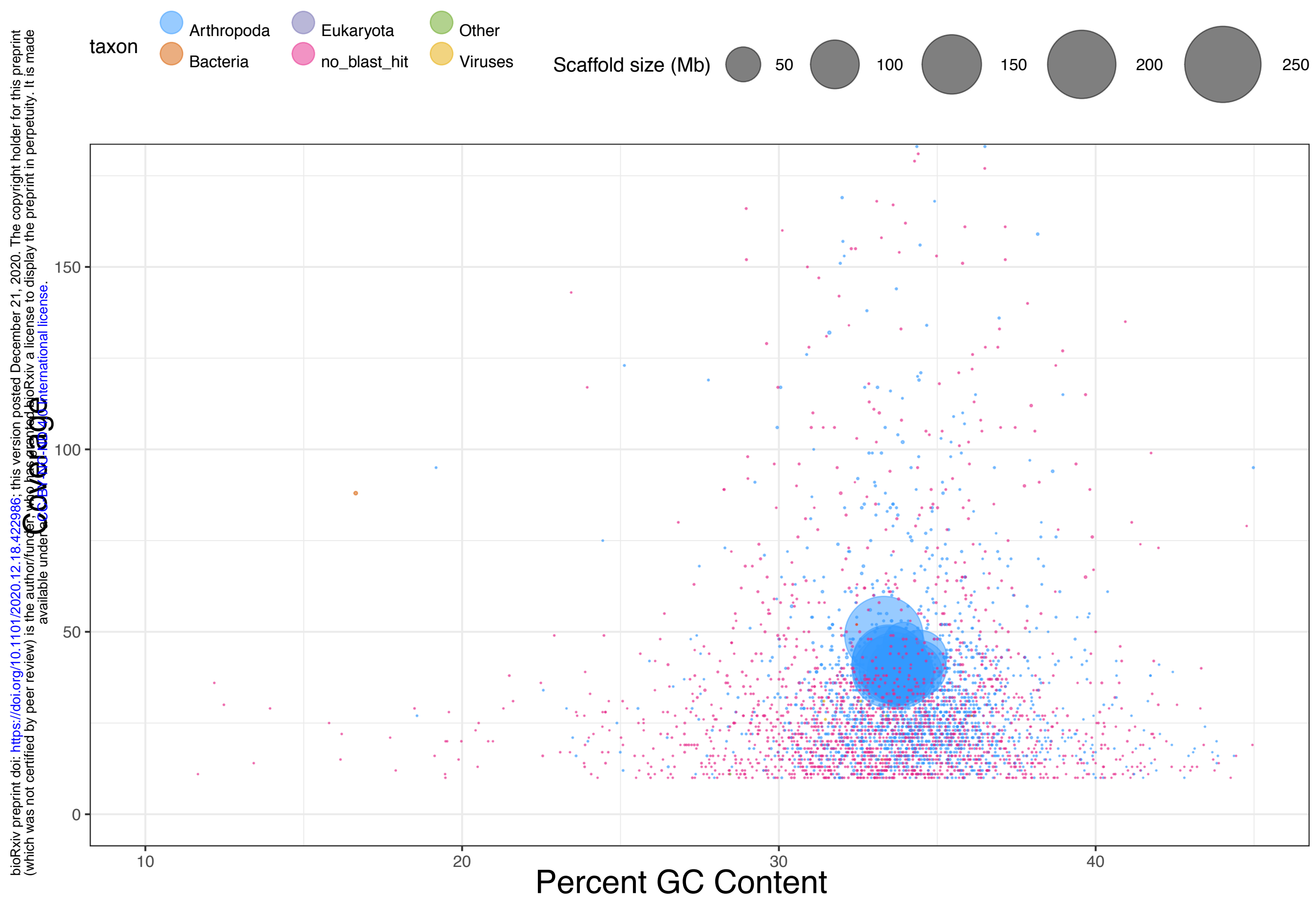




\section{Insecta BUSCO_V2}

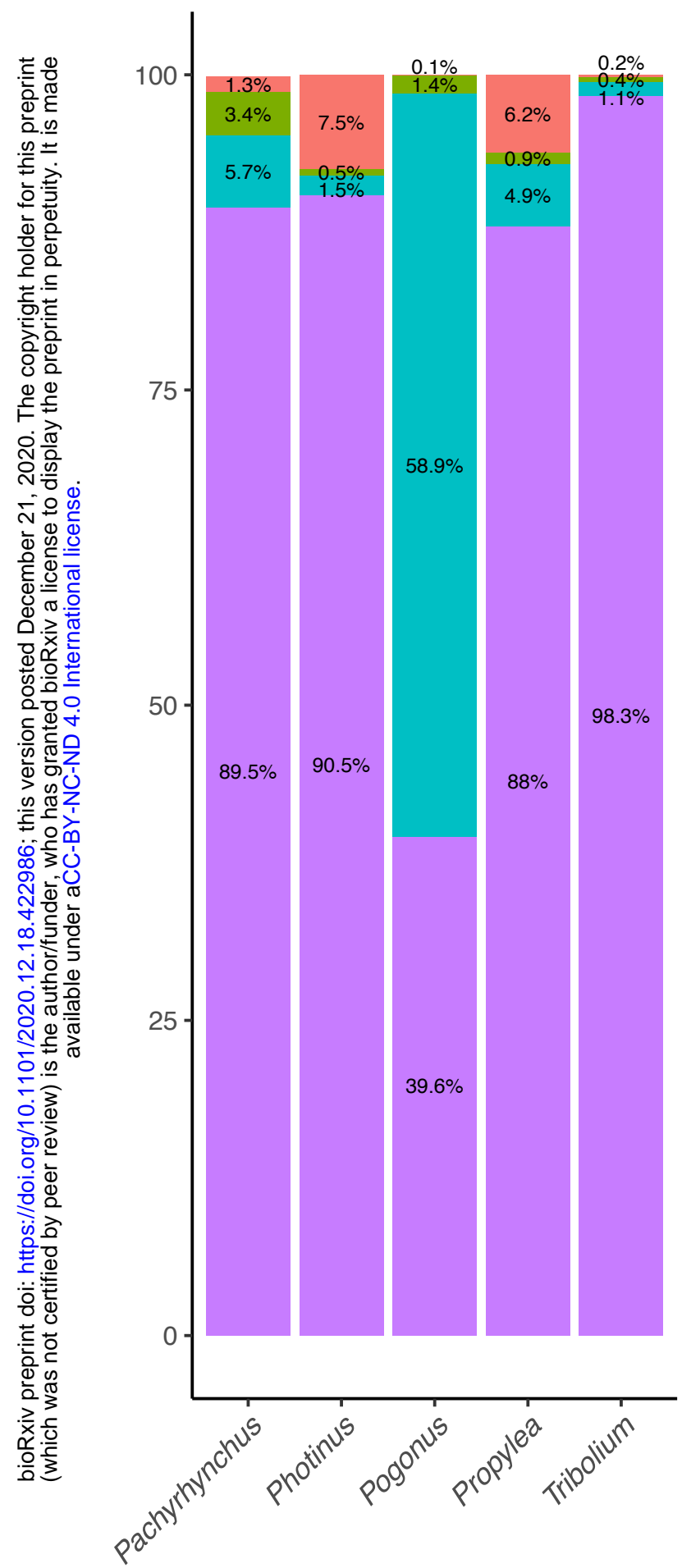

Insecta BUSCO_V4

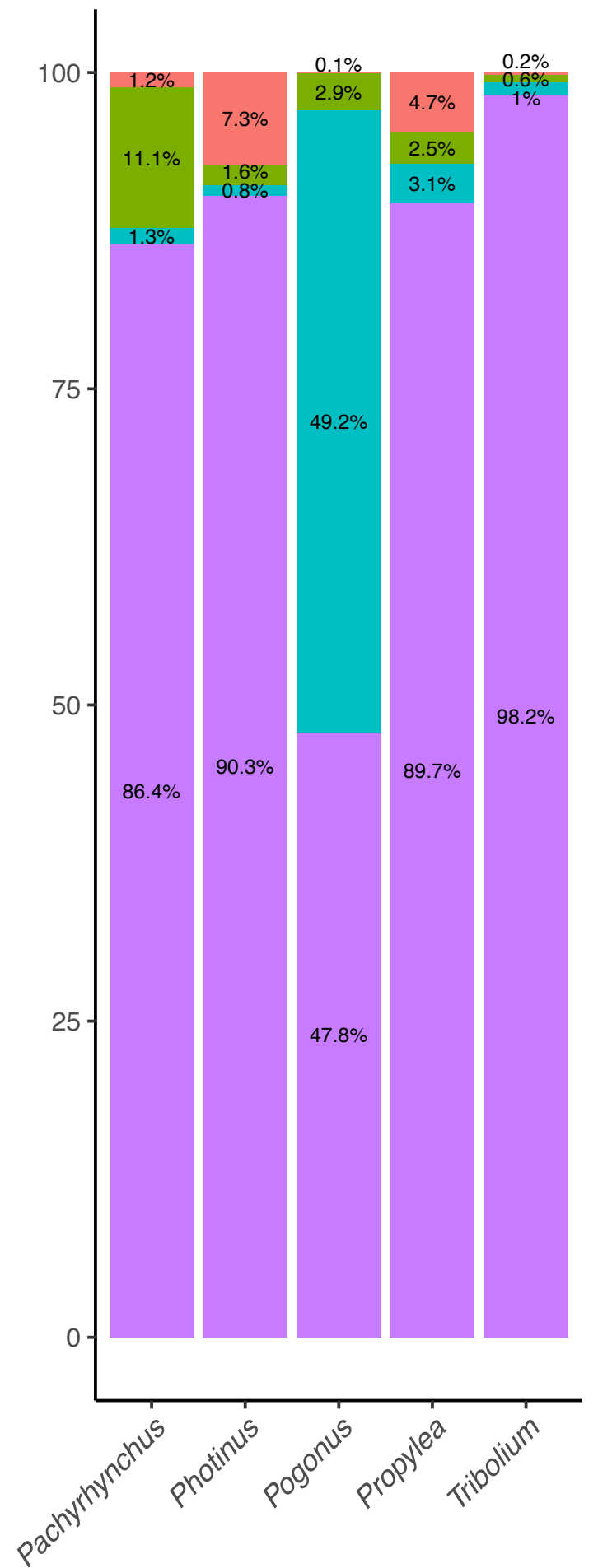

Insecta BUSCO_V5

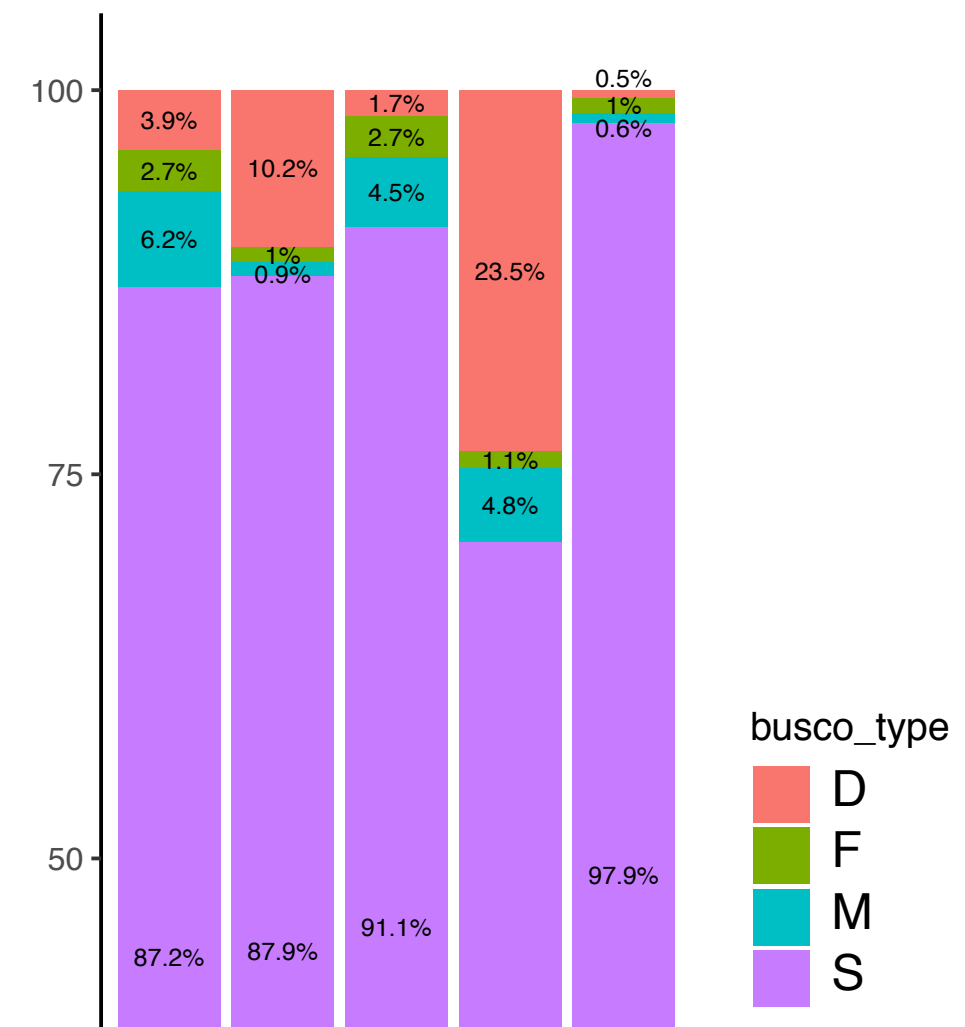


bioRxiv preprint doi: https://doi.org/10.1101/2020.12.18.422986; this version posted December 21, 2020. The copyright holder for this preprint (which was not certified by peer review) is the author/funder, who has granted bioRxiv a license to display the preprint in perpetuity. It is made Repeat Content

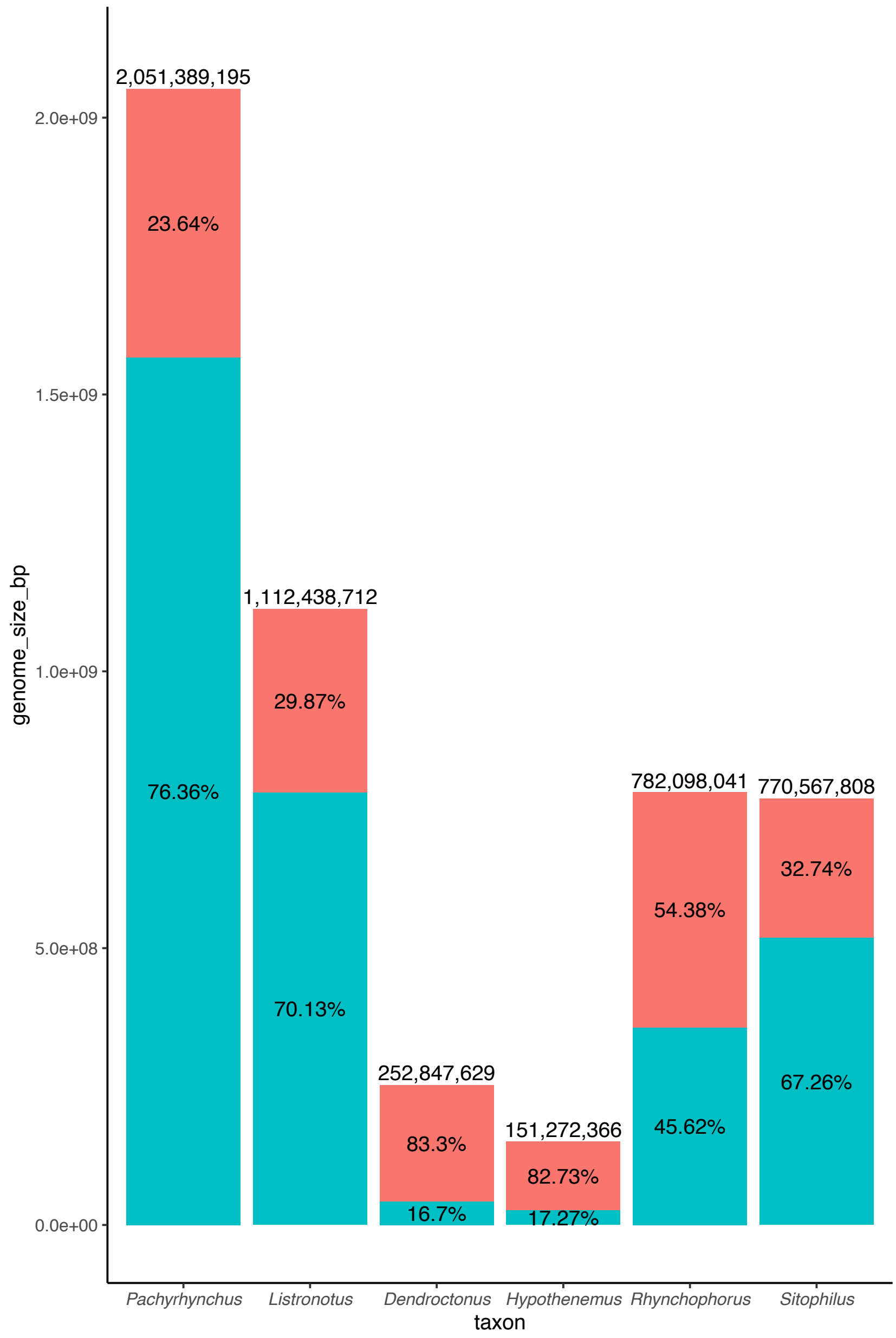


bioRxiv preprint doi: https://doi.org/10.1101/2020.12.18.422986; this version posted December 21, 2020. The copyright holder for this preprint

(which was not certified by peer review) is the author/funder, who has granted bioRxiv a license to display the preprint in perpetuity. It is made available under aCC-BY-NC-ND 4.0 International license.
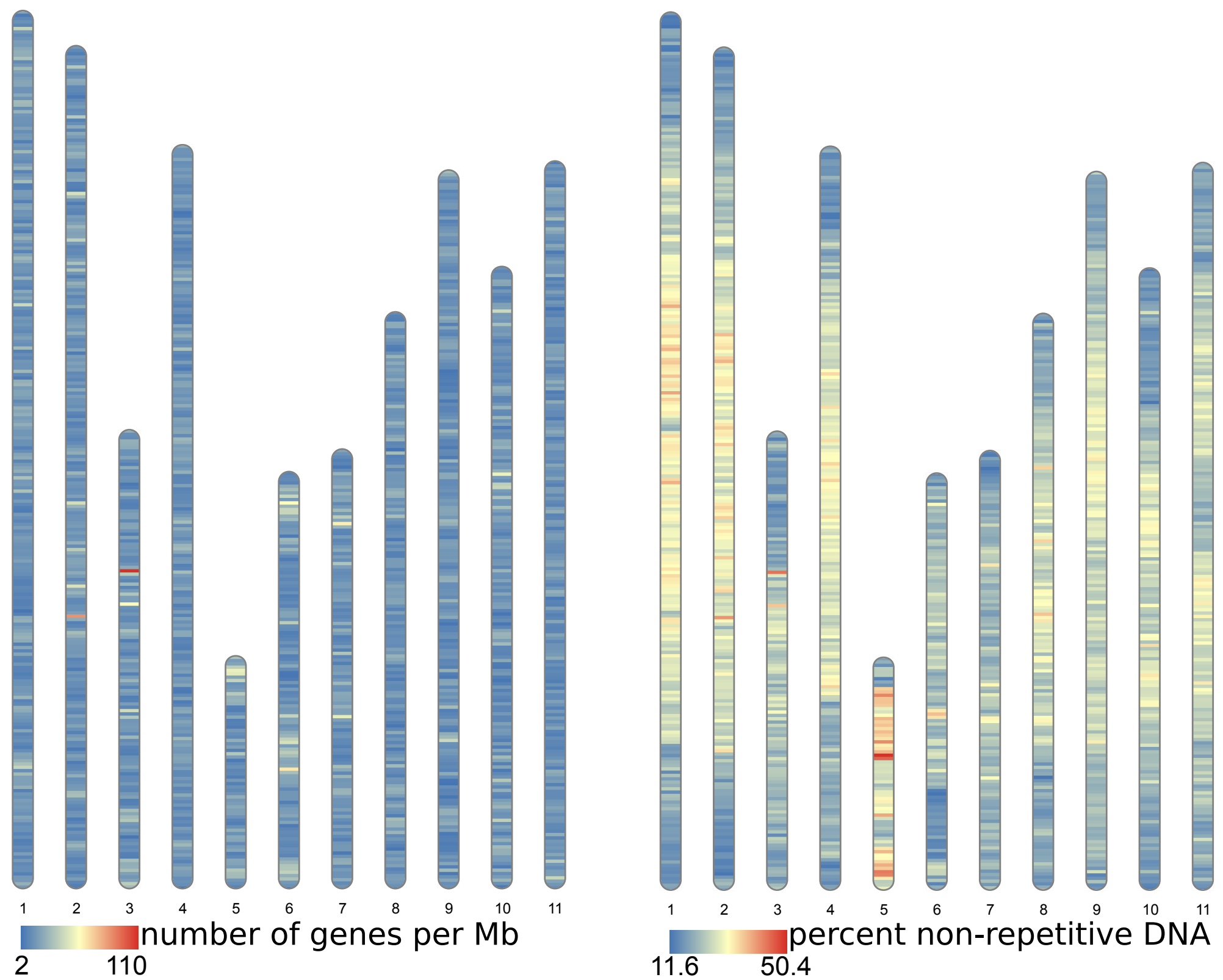

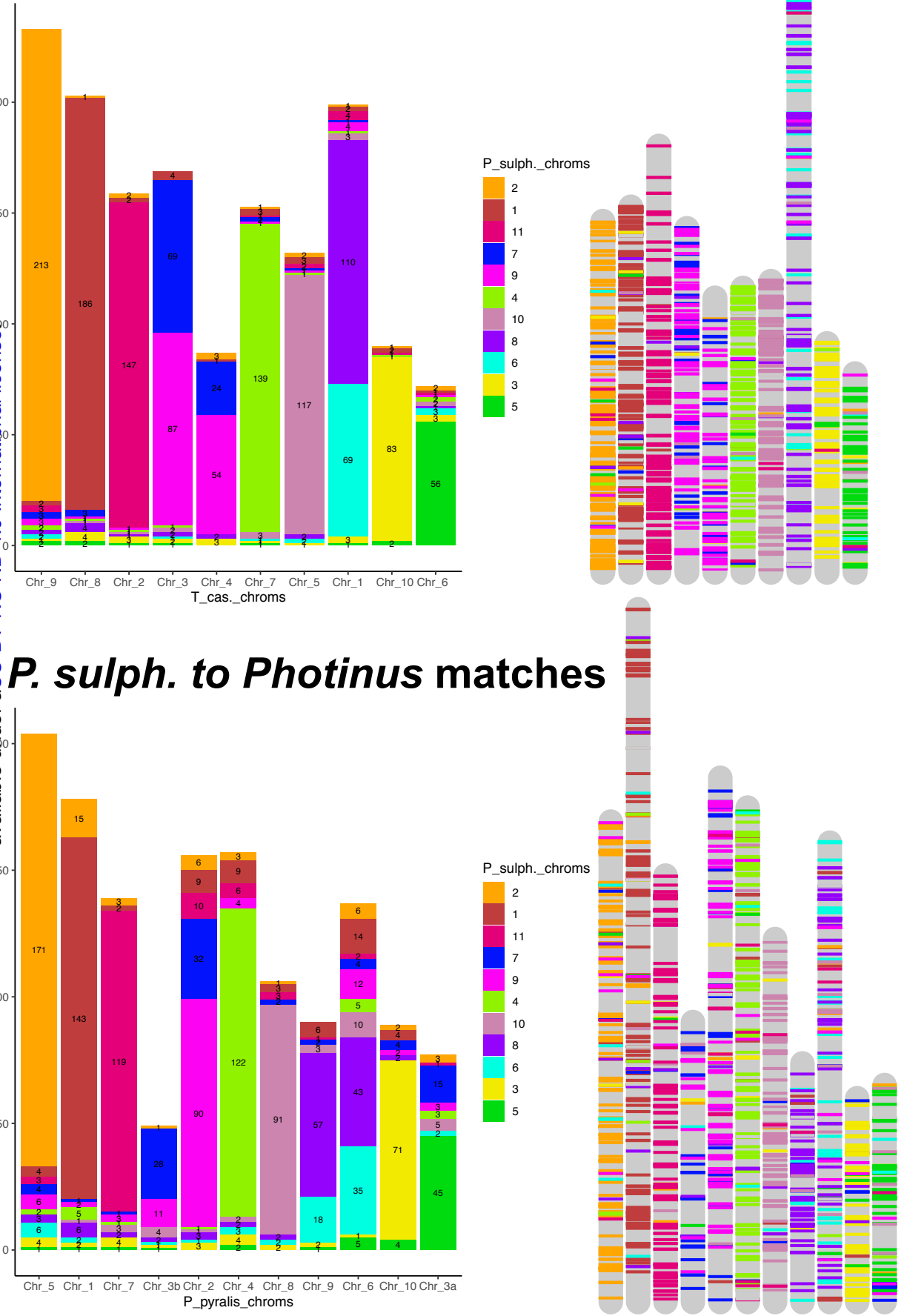

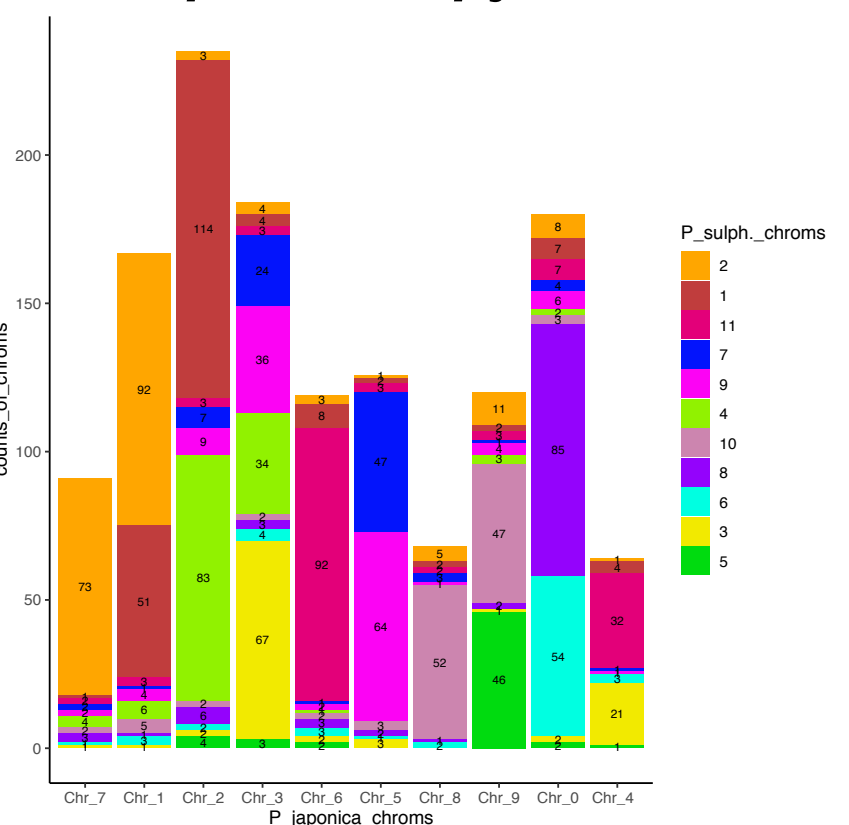

P. sulph. to Pogonus matches

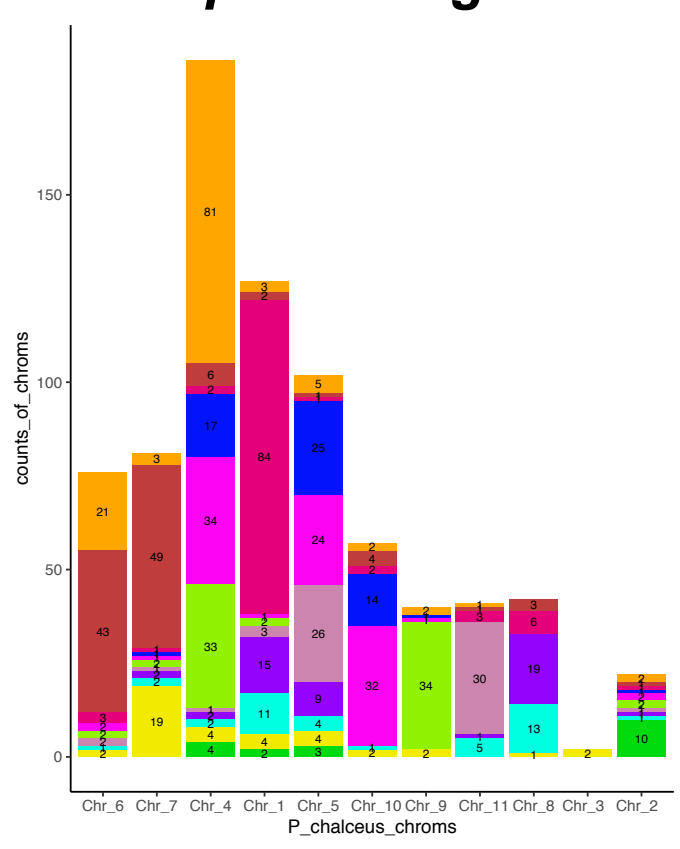

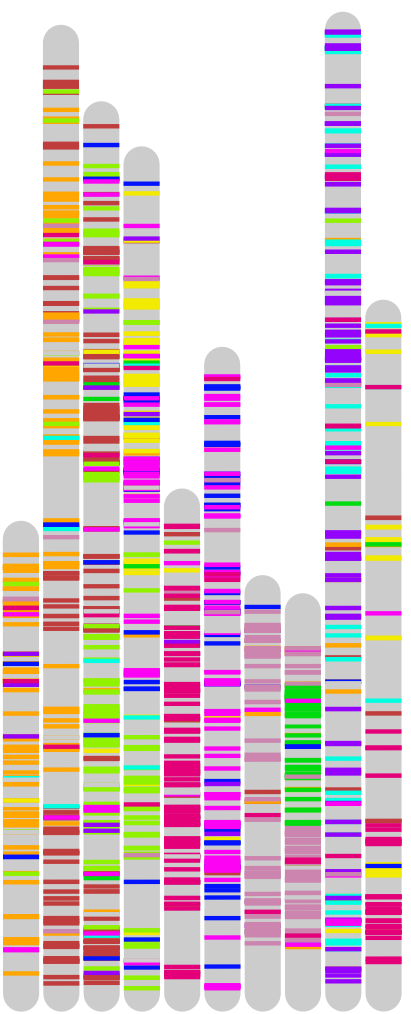

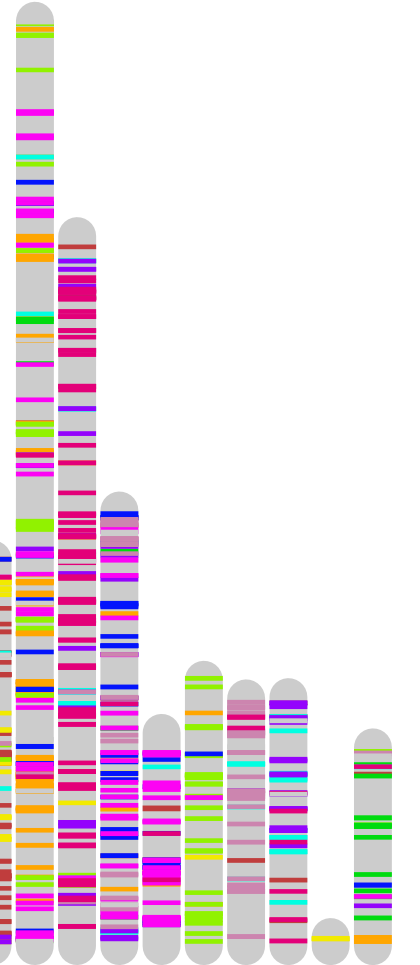




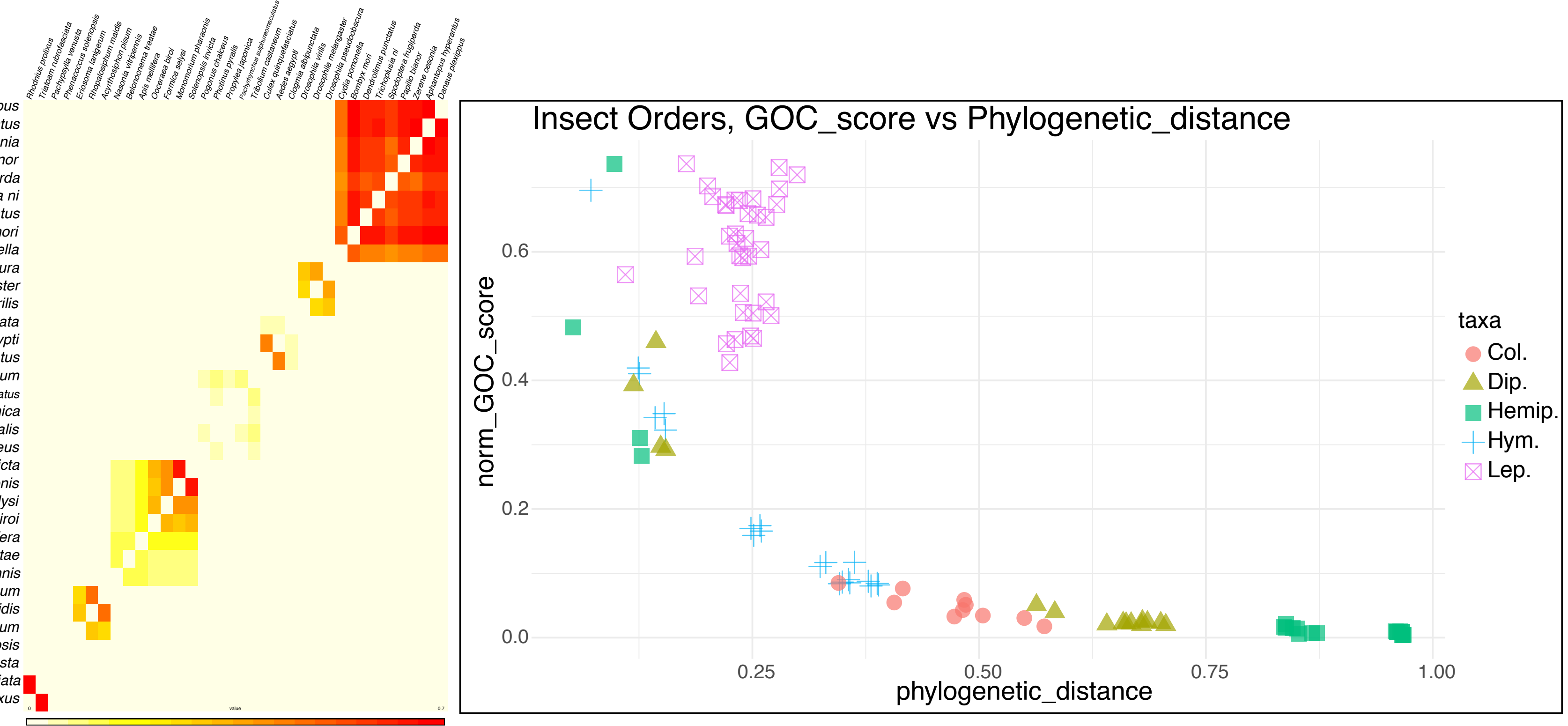

- Hemiptera Diptera

-Hymenoptera Coleoptera Lepidoptera 